\title{
Ergodicidade e homeomorfismos anulares do toro
}

\author{
Renato Belinelo Bortolatto
}

TESE APRESENTADA

$\mathrm{AO}$

Instituto de Matemática e Estatística

DA

Universidade de SÃo Paulo

PARA

OBTENÇÃO DO TÍTULO

$\mathrm{DE}$

Doutor EM CIÊNCIAS

\author{
Programa: Matemática Aplicada \\ Orientador: Prof. Dr. Fábio Armando Tal
}

Durante o desenvolvimento deste trabalho o autor recebeu auxílio financeiro do $\mathrm{CNPq}$

São Paulo, agosto de 2012 


\section{Ergodicidade e homeomorfismos anulares do toro}

Esta versão definitiva da tese contém as correções e alterações sugeridas pela Comissão Julgadora durante a defesa realizada por

Renato Belinelo Bortolatto em 22/06/2012.

Comissão Julgadora:

- Prof. Dr. Fábio Armando Tal (orientador) - IME-USP

- Prof. Dr. Salvador Addas Zanata - IME-USP

- Prof. Dr. Alejandro Kocsard - UFF

- Prof. Dr. Andrés Koropecki - UFF

- Prof. Dr. Mário Jorge Dias Carneiro - UFMG 


\section{Agradecimentos}

Quando eu conheci o Fábio ele já havia sido indicado como meu orientador e desde esse dia ele não foi nada menos do que generoso comigo. Ele provavelmente não faz idéia de quanto eu aprendi com ele, não apenas em matemática. Foi um privilégio e um prazer ter trabalhado ao lado dele esses anos. Obrigado por tudo, Fábio.

Quero também agradecer em poucas linhas o muito que as professoras Zara, Helena e Lucília acreditaram e investiram em mim durante minha gradução. Espero deixar vocês orgulhosas não só com o título, mas com tudo que o futuro trará.

Sendo aluno deste instituto por tanto tempo não posso deixar de ser grato pelos muitos amigos que conheci e que me fizeram sentir menos sozinho e mais feliz. Sou especialmente grato aqueles que foram meus professores e me ensinaram, inspiraram e ajudaram, cada um a sua maneira. Esses são muitos, mas em especial gostaria de agradecer a Mané, Salvador, Rosa, Gladys e Sérgio Oliva.

Por fim, agradeço minha família: Aline pela companhia e pela bagunça que fez no meu mundo ordenado e chato e meu pai por sempre ter feito por mim mais do que eu pedi. Mesmo sem dizer, espero que vocês todos saibam de minha gratidão em todos os dias que restam em nossas vidas. 


\section{Resumo}

Seja $f: \mathbb{T}^{2} \rightarrow \mathbb{T}^{2}$ um homeomorfismo homotópico a identidade e $F: \mathbb{R}^{2} \rightarrow$ $\mathbb{R}^{2}$ um levantamento de $f$ tal que seu conjunto de rotação $\rho(F)$ é um segmento vertical não degenerado contido em $\{0\} \times \mathbb{R}$.

Provamos que se $f$ é ergódico com respeito a medida de Lebesgue no toro e se o vetor de rotação médio (com respeito a mesma medida) é da forma $(0, \alpha)$ para $\alpha \in \mathbb{R} \backslash \mathbb{Q}$ então existe $M>0$ tal que $\left|\left(F^{n}(x)-x\right)_{1}\right| \leq M$ para todo $x \in \mathbb{R}^{2}$ e $n \in \mathbb{Z}$ (onde $(.)_{1}: \mathbb{R}^{2} \rightarrow \mathbb{R}$ é definida por $(x, y)_{1}=x$ ).

Palavras chave: Homeomorfismos do toro, conjuntos de rotação, ergodicidade, pontos periódicos. 


\begin{abstract}
Let $f: \mathbb{T}^{2} \rightarrow \mathbb{T}^{2}$ be a homeomorphism homotopic to the identity and $F$ : $\mathbb{R}^{2} \rightarrow \mathbb{R}^{2}$ a lift of $f$ such that the rotation set $\rho(F)$ is a non-degenerated vertical line segment contained in $\{0\} \times \mathbb{R}$.

We prove that if $f$ is ergodic with respect to the Lebesgue measure on the torus and the average rotation vector (with respect to same measure) is of the form $(0, \alpha)$ for $\alpha \in \mathbb{R} \backslash \mathbb{Q}$ then there exists $M>0$ such that $\left|\left(F^{n}(x)-x\right)_{1}\right| \leq M$ for all $x \in \mathbb{R}^{2}$ and $n \in \mathbb{Z}$ (where $(.)_{1}: \mathbb{R}^{2} \rightarrow \mathbb{R}$ is defined by $\left.(x, y)_{1}=x\right)$.
\end{abstract}

Key-words: Torus homeomorphisms, rotation sets, ergodicity, periodic points. 


\section{Sumário}

1 Introdução 1

2 Aplicações do círculo $\quad 3$

2.1 Número de rotação . . . . . . . . . . . . . . . . . . 3

2.2 Intervalo de rotação $\ldots \ldots \ldots \ldots$. . . . . . . . . 6

3 O conjunto de rotação 9

3.1 Definições . . . . . . . . . . . . . . . . . . . 9 9

3.2 Propriedades gerais dos conjuntos de rotação . . . . . . . . . 11

3.3 A geometria de um conjunto de rotação . . . . . . . . . . . . 13

3.4 Conjuntos de rotação e continuidade . . . . . . . . . . . . 15

3.5 Conjuntos de rotação com interior . . . . . . . . . . . 16

3.6 Conjuntos de rotação sem interior . . . . . . . . . . . . 17

4 A conjectura do deslocamento uniforme 21

4.1 Notação . . . . . . . . . . . . . . . . . . . . . 21

4.2 Motivação da conjectura . . . . . . . . . . . . . . . . 21

4.3 Nosso resultado . . . . . . . . . . . . . . . . . . . 23

4.4 O papel da ergodicidade . . . . . . . . . . . . . 24

5 Os conjuntos $B_{0}, B_{\pi}$ e $\omega\left(B_{0}\right), \omega\left(B_{\pi}\right) \quad \mathbf{2 7}$

5.1 Definições; $B_{0}, B_{\pi} \neq \emptyset \ldots \ldots \ldots \ldots \ldots . \ldots \ldots$



5.3 Estratégia da prova . . . . . . . . . . . . 33

6 Prova do teorema $\quad 37$

6.1 Consequências da negação do teorema . . . . . . . . . 37

6.2 O caso $\pi\left(\omega\left(B_{0}\right)\right) \cap \pi\left(\omega\left(B_{\pi}\right)\right) \neq \emptyset$ não pode ocorrer $\ldots . . .38$

6.3 A distância entre $\pi\left(\omega\left(B_{0}\right)\right)$ e $\pi\left(\omega\left(B_{\pi}\right)\right) \ldots \ldots . . . . . .40$

6.4 O caso $\pi\left(\omega\left(B_{0}\right)\right) \cap \pi\left(\omega\left(B_{\pi}\right)\right)=\emptyset$ não pode ocorrer $\ldots . . .42$

$\begin{array}{lll}7 & \text { Comentários finais } & 47\end{array}$ 


\section{Capítulo 1}

\section{Introdução}

O número de rotação $\rho(f) \in \mathbb{R}$ para homeomorfismos do círculo $\mathbb{T}^{1}$ que preservam a orientação é um invariante topológico bem conhecido cuja descoberta pode ser atribuida a Poincaré. Sabemos, por exemplo, que se $\rho(f) \notin \mathbb{Q}$ então existe uma semi-conjugação entre $f$ e a rotação de ângulo $\rho(f)$. O exemplo de Denjoy, por sua vez, mostra que em geral não é possível obter uma conjugação. No capítulo seguinte voltaremos a este assunto fazendo um breve resumo desta teoria.

Algumas vezes, no entanto, é preciso considerar não homeomorfismos, mas endomorfismos do círculo (isto é, aplicações contínuas de $\mathbb{T}^{1}$ de grau 1). Ainda no segundo capítulo apresentamos uma generalização adequada para o número de rotação devida a Newhouse, Palis e Takens [NPT83]. Vê-se facilmente que essa definição depende de cada ponto de $\mathbb{T}^{1}$, ao contrário do número de rotação de Poincaré. Olhando então para o fecho da união dos números de rotação pontuais obtemos um conjunto que mostra-se ser um intervalo fechado da reta, eventualmente degenerado. Chamamos assim este conjunto de intervalo de rotação de um endomorfismo do círculo.

Desejamos estudar aplicações contínuas do toro $m$-dimensional

$$
\mathbb{T}^{m} \cong \mathbb{R}^{m} / \mathbb{Z}^{m}
$$

nele mesmo (diremos simplesmente automorfismos do toro, quando a dimensão for fixada). Iremos pedir que essas aplicações sejam homotópicas a identidade pois queremos utilizar como ferramenta uma generalização apropriada do número de rotação para homeomorfismos do círculo. Veremos rapidamente que tal generalização será especialmente útil ao estudarmos as aplicações do toro 2-dimensional.

No terceiro capítulo definiremos então o conjunto de rotação para estes homeomorfismos e falaremos sobre suas propriedades e resultados pertinentes. Os resultados são usados para motivar a conjectura do deslocamento uniforme, que é explicada no quarto capítulo.

No quinto capítulo apresentamos as ferramentas que serão usadas na 
prova de um caso particular da conjectura do deslocamento uniforme no sexto capítulo.

No sétimo capítulo falamos sobre um resultado mais geral que pode ser obtido a partir de nossa prova e sobre perguntas que ficam em aberto. 


\section{Capítulo 2}

\section{Aplicações do círculo}

\subsection{Número de rotação}

Seja $f$ um homeomorfismo do círculo $\mathbb{T}^{1} \cong \mathbb{R} / \mathbb{Z}$ que preserva a orientação (com isso queremos dizer que consideramos uma ordem $\leq$ na reta e a descemos para $\preceq$ no círculo: " $f$ preserva a orientação" se $x \preceq y$ implica que $f(x) \preceq f(y))$. Naturalmente $\mathbb{T}^{1}$ é uma variedade, de forma que inicialmente pensamos em "descer" $f$ para $\mathbb{R}$. Contudo do ponto de vista da dinâmica será útil "levantar" $f$ para seu recobrimento universal como indica o diagrama abaixo

$$
\begin{array}{ccc}
\mathbb{R} & \stackrel{F}{\rightarrow} & \mathbb{R} \\
\downarrow \pi & & \downarrow \pi \\
\mathbb{T}^{1} & \stackrel{f}{\rightarrow} & \mathbb{T}^{1}
\end{array}
$$

onde $\pi: \mathbb{R} \rightarrow \mathbb{T}^{1}$ é definida por $\pi(x)=x(\bmod 1)$. Como $\pi$ não é invertível o diagrama acima define apenas uma semi-conjugação entre $f$ e seu levantamento $F$. O levantamento não é único e por isso sempre precisaremos escolher um. Apesar disso vê-se facilmente no diagrama acima que se $F_{1}$ e $F_{2}$ são dois levantamentos de $f$ então $F_{1}-F_{2} \in \mathbb{Z}$.

Também é fácil ver que $F(x+i)=F(x)+i$ para todo $i \in \mathbb{Z}$ e neste caso dizemos que $F$ é uma aplicação de grau 1. Observe que apesar de $f$ e $F$ serem semi-conjugados essas aplicações não possuem, em geral, a mesma dinâmica: basta considerar em $\mathbb{T}^{1} \simeq \mathbb{R} / \mathbb{Z}$ a aplicação

$$
r_{\frac{1}{4}}(\theta)=\theta+\frac{1}{4}
$$

(para o qual todos os pontos são periódicos) e seu levantamento

$$
R_{\frac{1}{4}}(x)=x+\frac{1}{4}
$$

que não tem pontos periódicos. 
É interessante notar que se $f$ inverte a orientação (i.e., se $x \preceq y$ então $f(x) \succeq f(y))$ pode-se provar que $f$ tem exatamente 2 pontos fixos. Desta forma o caso mais interessante do ponto de vista dinâmico é dos homeomorfismos que preservam orientação: esssa hipótese será usada fortemente no restante dessa seção.

Definição 1. Denotaremos por $h_{+}\left(\mathbb{T}^{1}\right)$ o conjunto dos homeomorfismos de $\mathbb{T}^{1}$ que preservam a orientação.

Definição 2. Seja $f \in h_{+}\left(\mathbb{T}^{1}\right)$ e $F$ um levantamento. Quando existir o limite

$$
\tau(F, x):=\lim _{n \rightarrow \infty} \frac{F^{n}(x)}{n}
$$

diremos que $\tau(F, x)$ é o número de translação de $x \in \mathbb{R}$ com respeito a $F$.

Proposição 1. Seja $f \in h_{+}\left(\mathbb{T}^{1}\right)$ e $F$ um levantamento. Então

1. Existe $\lim _{n \rightarrow \infty} \frac{F^{n}(x)}{n}$ para todo $x \in \mathbb{R}$.

2. $\tau(F, x)=\tau(F)$, isto é, $\tau(F, x)$ independe de $x \in \mathbb{R}$.

3. Se $f$ tem um ponto periódico então $\tau(F) \in \mathbb{Q}$.

Note que se $F_{1}, F_{2}$ são dois levantamentos de $f$ então $\tau\left(F_{1}\right)-\tau\left(F_{2}\right) \in \mathbb{Z}$. Isso permite definir o número de rotação diretamente para um homeomorfismo do círculo que preserva orientação.

Definição 3. Se $f \in h_{+}\left(\mathbb{T}^{1}\right)$ definimos o número de rotação de $f$ como

$$
\rho(f)=\pi(\tau(F))
$$

Quando não houver confusão indentificamos $\rho(f) \in \mathbb{T}^{1}$ com a parte nãointeira de $\tau(F)$. Observe então que $\rho(f) \in[0,1[$.

O número de rotação possui duas propriedades interessantes que descrevemos nos dois enunciados seguintes.

Proposição 2. Se $f, g \in h_{+}\left(\mathbb{T}^{1}\right)$ são topologicamente conjugadas $\left(e m h_{+}\left(\mathbb{T}^{1}\right)\right.$ ) então $\rho(f)=\rho(g)$.

Proposição 3. Seja $f \in h_{+}\left(\mathbb{T}^{1}\right)$. Então $\rho(f) \notin \mathbb{Q}$ se e somente se $f$ não tem pontos periódicos.

Uma pergunta natural nesse ponto é quando um homeomorfismo do círculo que preserva a orientação é conjugado a uma rotação. No caso de uma rotação por ângulo racional (que claramente possui número de rotação racional) todas as órbitas são periódicas e de mesmo período; por outro lado se $f \in h_{+}\left(\mathbb{T}^{1}\right)$ e $\rho(f) \in \mathbb{Q}$ a proposição anterior garante apenas a existência de uma órbita periódica. Pode-se ver também que todas as órbitas periódicas 
possuem o mesmo período e estão ordenadas como a rotação de ângulo $\rho(f)$. Não é difícil construir (começando com alguns pontos periódicos e fazendo as outras órbitas serem heteroclínicas a estes pontos periódicos) uma infinidade de aplicações em $h_{+}\left(\mathbb{T}^{1}\right)$ que possuem número de rotação racional mas não podem ser conjugadas a uma rotação.

Neste sentido, o caso de uma rotação por ângulo irracional é mais interessante. Temos, por exemplo, o seguinte resultado.

Proposição 4. Seja $f \in h_{+}\left(\mathbb{T}^{1}\right)$ e suponha que $\rho(f) \notin \mathbb{Q}$. Então existe $h: \mathbb{T}^{1} \rightarrow \mathbb{T}^{1}$ contínua, sobrejetora, que preserva ordem e tal que

$$
h \circ f=R_{\rho(f)} \circ h
$$

isto é, f é semi-conjugada a uma rotação de ângulo irracional.

Pode-se provar ainda que se $f$ é topologicamente transitiva então a semiconjugação no teorema acima é uma conjugação. Note porém que existe uma aplicação do círculo que possui número de rotação irracional mas que não é conjugada a uma rotação irracional.

Exemplo (Denjoy). Começe com uma rotação irracional e tome uma órbita. Mostra-se que trocando os pontos dessa órbita por intervalos $I_{n}$ suficientemente pequenos (que não cobrem $\mathbb{T}^{1}$ ) obtem-se uma aplicação $g \in C^{1}\left(\mathbb{T}^{1}\right)$ que satisfaz $g\left(I_{n}\right)=I_{n+1}$ e $g_{\mid\left(\cup_{n \in \mathbb{Z}} I_{n}\right)}$ é semi-conjugada a uma rotação irracional.

Observe então que para uma aplicação $f$ do círculo ser conjugada a uma rotação irracional é preciso que $f$ tenha todas as órbitas densas em $\mathbb{T}^{1}$. Isso mostra que $g$ não pode ser conjugada a uma rotação irracional.

É possivel construir esse exemplo de forma que $g \in C^{1+\varepsilon}$ para $0 \leq \varepsilon<$ 1 e de forma que a medida de Lebesgue de $\cup_{n \in \mathbb{Z}} I_{n}$ seja qualquer número estritamente positivo menor que 1.

O teorema de Denjoy diz que se uma aplicação do círculo que preserva orientação, possui número de rotação irracional, é $C^{1}$ e sua derivada tem variação limitada então ela é transitiva (e portanto conjugada a uma rotação). Em particular, toda aplicação $C^{2}$ que preserva orientação possui número de rotação irracional é conjugada a uma rotação por ângulo irracional.

Para finalizar essa seção citamos dois resultados que terão paralelos no próximo capítulo.

Proposição 5 ([KH96], Theorem 11.2.9). Se f é um homeomorfismo que preserva orientação do círculo com $\rho(f) \notin \mathbb{Q}$ então $f$ é unicamente ergódico. Visto como uma transformação que preserva medida, $f$ é métricamente isomorfa a uma rotação irracional.

Corolário ([KH96], Corollary 11.2.10). Um homeomorfismo do círculo que que preserva orientação possui entropia topológica zero. 
Voltamos a notar que nessa seção a propriedade de preservação da orientação foi decisiva. Entretanto veremos a seguir que podemos, de certa forma, abrir mão desta hipótese e ainda obter resultados interessantes. Isso indica a possibilidade de que uma versão adequada do número de rotação dos homeomorfismo do círculo que preservam a orientação seja útil no estudo da dinâmica em variedades de dimensão maior do que um.

\subsection{Intervalo de rotação}

O conceito de intervalo de rotação para endomorfismos do círculo aparece em [NPT83] motivado por um problema em teoria das bifurcações. As proposições e provas abaixo são da mesma fonte.

Denote por $\operatorname{End}\left(\mathbb{T}^{1}\right)$ o conjunto das aplicações contínuas $\phi: \mathbb{T}^{1} \rightarrow \mathbb{T}^{1}$ de grau 1. Para $\phi \in \operatorname{End}\left(\mathbb{T}^{1}\right)$, um levantamento $\Phi$ e um ponto $x \in \mathbb{T}^{1}$ definimos o número de rotação puntual

$$
\rho(\Phi, x):=\limsup _{n \rightarrow \infty} \frac{\Phi^{n}(x)-x}{n}
$$

Definimos o conjunto de rotação por

$$
\rho(\Phi):=\overline{\bigcup_{x \in \mathbb{T}^{1}} \rho(\Phi, x)}
$$

Tanto o número de rotação puntual como o conjunto de rotação são invariantes topológicos. Note que, para um homeomorfismo do círculo que preserva a orientação, o conjunto de rotação coincide com o número de rotação.

O conjunto de rotação é fechado por definição. Queremos mostrar que ele é um ponto ou um intervalo da reta. Temos o seguinte lema:

Lema 1 ([NPT83], Lemma (3.1)). Suponha que $\phi \in \operatorname{End}\left(\mathbb{T}^{1}\right)$ e seja $\Phi$ um levantamento. Se $\phi$ não tem um ponto periódico com número de rotação puntual $\frac{p}{q}$ com $p \in \mathbb{Z}$ e $q \in \mathbb{N}$ então $\rho(\Phi)$ está contido em

$$
\left\{x \in \mathbb{R} \mid x<\frac{p}{q}\right\} \quad \text { ou } \quad\left\{x \in \mathbb{R} \mid x>\frac{p}{q}\right\}
$$

Demonstração. Observe que, para todo $x \in \mathbb{R}$, temos $\Phi^{q}(x)-x \neq p$ pois, caso contrário, $\pi(x)$ seria um ponto periódico de $\phi$ com número de rotação puntual $\frac{p}{q}$. Note que

$$
\Phi^{q}(x+1)-(x+1)=\Phi^{q}(x)-x
$$

isto é, $\Phi^{q}(x)-x$ é uma função periódica (em $x$ ) de período 1. Assim, sendo contínua e podendo ser entendida com domínio [0,1], existe um $\varepsilon>0$ tal que

$$
\Phi^{q}(x)-x<p-\varepsilon \quad \text { ou } \quad \Phi^{q}(x)-x>p+\varepsilon
$$


para todo $x \in \mathbb{R}$, ou seja,

$$
\rho(\Phi) \subseteq\left\{x \in \mathbb{R} \mid x<\frac{p}{q}\right\} \quad \text { ou } \quad \rho(\Phi) \subseteq\left\{x \in \mathbb{R} \mid x>\frac{p}{q}\right\}
$$

Corolário ([NPT83], Corollary (3.2)). Seja $\phi \in \operatorname{End}\left(\mathbb{T}^{1}\right)$ e $\Phi$ um levantamento. Suponha que $\alpha, \beta \in \rho(\Phi)$ e que existe $\frac{p}{q} \in \mathbb{Q}$ satisfazendo $\alpha \leq \frac{p}{q} \leq \beta$. Então $\phi$ tem um ponto periódico com número de rotação puntual $\frac{p}{q}$.

Demonstração. Se $\phi$ não tem um ponto periódico com número de rotação puntual $\frac{p}{q}$ então pelo lema anterior que

$$
\rho(\Phi) \subseteq\left\{x \in \mathbb{R} \mid x<\frac{p}{q}\right\} \quad \text { ou } \quad \rho(\Phi) \subseteq\left\{x \in \mathbb{R} \mid x>\frac{p}{q}\right\}
$$

Mas isso não pode acontecer pois $\alpha, \beta \in \rho(\Phi)$ e $\alpha \leq \frac{p}{q} \leq \beta$.

Corolário. O conjunto de rotação de um levantamento $\Phi$ de um endomorfismo $\phi: \mathbb{T}^{1} \rightarrow \mathbb{T}^{1}$ é um ponto ou um intervalo fechado da reta. 


\section{Capítulo 3}

\section{O conjunto de rotação}

\subsection{Definições}

Seja $f: \mathbb{T}^{m} \rightarrow \mathbb{T}^{m}$ e $F: \mathbb{R}^{m} \rightarrow \mathbb{R}^{m}$ um levantamento fixado. Seja $\pi: \mathbb{R}^{2} \rightarrow$ $\mathbb{T}^{2}$ a projeção natural. Uma forma natural de definir o vetor de rotação para $p \in \mathbb{T}^{m}$ seria tomar $x \in \pi^{-1}(p)$ e considerar $\rho(F, x)=\lim _{n \rightarrow \infty} \frac{F^{n}(x)-x}{n}$. Definimos então o conjunto de rotação baseado em pontos como

$$
\rho_{p}(F)=\cup_{x \in \mathbb{R}^{m}} \rho(F, x)
$$

Em tempo, é necessário nos perguntarmos se $\rho_{p}(F)$ está bem definido. Para isso usamos o seguinte resultado.

Lema 2. Um homeomorfismo $F$ do $\mathbb{R}^{m}$ é um levantamento de um homeomorfismo de $\mathbb{T}^{m}$ homotópico a identidade se, e só se, $F(x+v)=F(x)+v$ para todo $x \in \mathbb{R}^{m}$ e $v \in \mathbb{Z}^{m}$.

A demonstração desse lema não é difícil, mas necessita de um resultado de topologia algébrica que nos desviaria de nosso caminho (uma prova pode ser encontrada em [BLR07]). Ao assumirmos esse resultado é imediato que, se $f$ é homotópica a identidade, $\rho_{p}(F)$ está bem definido e $\rho_{p}(F)=\cup_{x \in[0,1]^{m}} \rho(F, x)$.

Evidentemente, quando $m=1$ e $f$ preserva orientação, $\rho_{p}(F)$ coincide com o número de rotação. Contudo, para $m \geq 2, \rho(F, x)$ pode depender de $x$ e de forma a não possuir em geral boas propriedades topológicas. De fato, mesmo quando $F$ é levantamento de um automorfismo do toro 2-dimensional, homotópico à identidade, o conjunto $\rho_{p}(F)$ não precisa ser convexo, nem mesmo conexo.

Exemplo. Considere um fluxo do tipo Reeb em $\mathbb{R}^{2}$. Esse fluxo é mais facilmente explicado por um desenho (veja abaixo). Podemos construir um exemplo em alguns passos, começando definindo sobre as retas $]-\infty,+\infty[\times\{0\}$, 


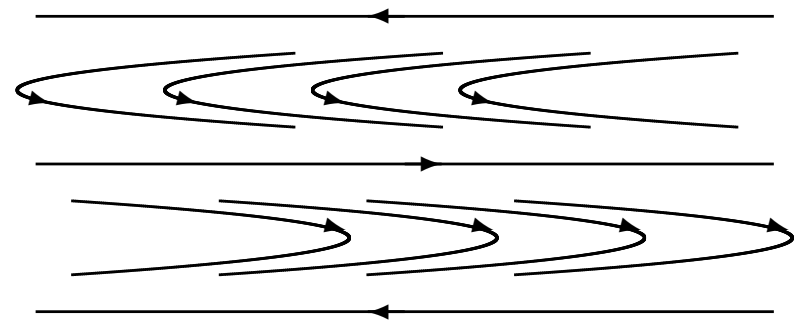

Figura 3.1: Fluxo de Reeb representado na faixa $]-\infty,+\infty[\times[0,1]$.

]$-\infty,+\infty\left[\times\left\{\frac{1}{2}\right\}\right.$ e $]-\infty,+\infty[\times\{1\}$ um campo v que vale $(-1,0),(1,0) e$ $(-1,0)$, respectivamente.

Estendemos então o campo em $]-\infty,+\infty[\times[0,1]$ de forma que as curvas integrais restantes sejam assintóticas as retas dadas anteriormente $e$ satisfaçam $v(x+1, y)=v(x, y)$ e $v\left(x, y+\frac{1}{2}\right)=-v(x, y)$. Estendemos esse campo para todo plano de forma natural e consideramos o homeomorfismo dado pelo fluxo no tempo 1.

Pelo lema 2 esse homeomorfismo é um levantamento de um homeomorfismo do toro homotópico a identidade. Além disso, como a órbita de qualquer ponto tende a uma das retas $\mathbb{R} \times \frac{1}{2} \mathbb{Z}$, pode-se ver que $\rho_{p}(F)=$ $\{(1,0)\} \cup\{(-1,0)\}$.

Note que no exemplo acima tomar o fecho topológico de $\rho_{p}(F)$ (como foi feito para o intervalo de rotação) nada modifica. Podemos, é claro, considerar o fecho convexo de $\rho_{p}(F)$, que denotaremos $\operatorname{Conv}\left(\rho_{p}(F)\right)$. O conjunto $\operatorname{Conv}\left(\rho_{p}(F)\right)$ é um bom candidato para conjunto de rotação (ao menos coincide com um intervalo nesse exemplo), mas não é, a priori, nossa única opção.

Outra possibilidade é definir como conjunto de rotação

$$
\rho(F)=\bigcap_{n \geq 1} \overline{\bigcup_{k \geq n}\left\{\frac{F^{k}(x)-x}{k}: x \in I^{m}\right\}}
$$

onde $I^{m}:=[0,1]^{m}$ (vide o lema anterior). Esta definição lembra a noção de $\omega$-limite e portanto é também natural por tentar capturar o comportamento assintótico das órbitas de forma semelhante ao número de rotação de Poincaré. Notamos que $\rho(F)$ é igual ao conjunto dos pontos de acumulação das sequências

$$
\rho_{k}\left(F, x_{n_{k}}\right):=\frac{F^{k}\left(x_{n_{k}}\right)-x_{n_{k}}}{k}
$$

para $x_{n} \in \mathbb{R}^{m}$ com $n, k \in \mathbb{N}$. Segue que para $m=1$ a definição que demos para o conjunto de rotação também coincide com o número de rotação. Note 
que $\rho(F)$ é fechado por definição. Pode-se ver que ele é conexo e que, se $m=2$, também é convexo [MZ89].

Podemos ainda apontar mais uma possível definição de conjunto de rotação em $\mathbb{T}^{m}$. Considere $\mathcal{M}(f)$ o espaço de todas as medidas de probabilidade, $f$-invariantes em $\mathbb{T}^{m}$ e $\mathcal{M}_{E}(f)$ o subsespaço das medidas ergódicas de $\mathcal{M}(f)$. O conjunto $\mathcal{M}(f)$ é compacto se considerado com a topologia *-fraca (mediante a identificação das medidas com funcionais lineares em $\mathbb{C})$.

Seja $\mu \in \mathcal{M}_{E}(f)$. Pelo teorema de Birkhoff temos para $\mu$-qtp $x \in X$ que

$$
\frac{1}{n} \sum_{i=0}^{n-1} \phi \circ f^{k} \stackrel{n \rightarrow \infty}{\longrightarrow} \int \phi d \mu
$$

para toda aplicação contínua $\phi: \mathbb{T}^{m} \rightarrow \mathbb{R}^{m}$. Considere em particular a aplicação $\phi(x)=F(y)-y$, onde $y \in \pi^{-1}(x)$ (que está bem definida pois $F$ comuta com as translações por vetores de $\left.\mathbb{Z}^{2}\right)$. Temos então que

$$
\frac{1}{n} \sum_{i=0}^{n-1} \phi \circ f^{k}(x)=\frac{1}{n} \sum_{i=0}^{n-1}\left(F^{k+1}(y)-F^{k}(y)\right)=\frac{F^{n}(y)-y}{n}
$$

Concluímos que para $\mu$-qtp $x \in \mathbb{T}^{m}$ e todo $y \in \pi^{-1}(x)$

$$
\frac{F^{n}(y)-y}{n} \stackrel{n \rightarrow \infty}{\longrightarrow} \int \phi d \mu
$$

ou seja, $\int \phi d \mu \in \rho_{p}(F)$ para todo $\mu \in \mathcal{M}_{E}(f)$. Definimos

$$
\rho_{\text {erg }}(F)=\left\{\int \phi d \mu: \mu \in \mathcal{M}_{E}(f)\right\}
$$

Como os pontos extremais (no sentido da análise convexa) de $\mathcal{M}(f)$ são justamente as medidas em $\mathcal{M}_{E}(f)$, temos que o fecho convexo de $\rho_{\text {erg }}(F)$ é

$$
\operatorname{Conv}\left(\rho_{\text {erg }}(F)\right)=\left\{\int \phi d \mu: \mu \in \mathcal{M}(f)\right\}
$$

Definimos $\rho_{\text {mes }}(F):=\operatorname{Conv}\left(\rho_{\text {erg }}(F)\right)$.

\subsection{Propriedades gerais dos conjuntos de rotação}

Como as três definições da seção anterior tem boas razões de ser, uma boa estratégia é estudarmos de que forma elas estão relacionadas.

Proposição 6 ([MZ89], Corollary 2.4). Seja F um levantamento de um homeomorfismo $f$ de $\mathbb{T}^{2}$ então

$$
\operatorname{Conv}\left(\rho_{p}(F)\right)=\rho(F)=\rho_{\text {mes }}(F)
$$


Em contraste, temos, para $\mathbb{T}^{3}$ um exemplo em [LM91] que mostra que $\operatorname{Conv}\left(\rho_{p}(F)\right) \neq \rho(F)$. Esse é um dos motivos porque iremos estudar apenas o caso $m=2$. No entanto,

$$
\rho_{\text {mes }}(F)=\operatorname{Conv}(\rho(F))
$$

para qualquer $F$ que seja levantamento de uma aplicação contínua $f$ do toro $m$-dimensional, homotópica à identidade ([MZ89], Theorem 2.4). Assim, nas mesmas hipóteses, temos para $\mathbb{T}^{m}$ que

$$
\operatorname{Conv}\left(\rho_{p}(F)\right)=\operatorname{Conv}(\rho(F))
$$

e que

$$
\operatorname{Conv}(\rho(F))=\operatorname{Conv}\left(\cup_{x \in \pi^{-1}(\Omega(f))} \rho(F, x)\right)
$$

onde $\Omega(f)$ é o conjunto dos pontos não-errantes de $f$ (o que é consequência de que, com respeito a uma medida $\mu$, quase todo ponto é não-errante). Essa última igualdade é útil para determinar o conjunto de rotação de alguns exemplos explícitos.

Os conjuntos de rotação em $\mathbb{T}^{m}$ possuem algumas propriedades que são úteis para descrever o conjunto de rotação de seus diferentes levantamentos e o conjunto de rotação das iteradas de $f$.

Proposição 7 ([MZ89]). Seja F um levantamento de uma aplicação contínua $f$ do toro $m$-dimensional, homotópica à identidade. Então para todo $p \in \mathbb{Z}^{m}$ e $n \in \mathbb{N}$ temos que

- $\rho\left(F^{q}-p\right)=q \rho(F)-p$

- $\rho\left(F^{q}-p, x\right)=q \rho(F, x)-p$, para todo $x \in \mathbb{R}^{m}$

- $\rho_{p}\left(F^{q}-p\right)=q \rho_{p}(F)-p$

- $\rho_{\text {mes }}\left(F^{q}-p\right)=q \rho_{\text {mes }}(F)-p$

Além disso, se f é um homeomorfismo então

- $\rho\left(F^{-1}\right)=-\rho(F)$

- $\rho_{\text {mes }}\left(F^{-1}\right)=-\rho_{\text {mes }}(F)$

Apesar de simples, essas propriedades podem ser bastante úteis e serão de fato usadas no restante da tese. Vejamos um importante exemplo de seu uso a seguir. Assumiremos o seguinte resultado:

Proposição 8 ([Fra88], Theorem 3.5). Seja $F$ um levantamento de um homeomorfismo $f$ de $\mathbb{T}^{2}$, homotópico à identidade. Se $0 \in \rho_{\text {erg }}(F)$ então $F$ tem um ponto fixo. 
Provemos então a seguinte proposição.

Proposição 9 ([MZ89], Theorem 3.10). Seja F um levantamento de um homeomorfismo $f$ de $\mathbb{T}^{2}$, homotópico à identidade. Suponha que $(r / q, s / q) \in$ $\rho_{\text {erg }}(F)$ para $r, s \in \mathbb{Z}, q \in \mathbb{N}$ com $m d c(r, s, q)=1$. Então existe um ponto f-periódico $x \in \mathbb{T}^{2}$ de período mínimo $q$ e que satisfaz $\rho(F, y)=\left\{\left(\frac{r}{q}, \frac{s}{q}\right)\right\}$ para todo $y \in \pi^{-1}(x)$.

Demonstração. Por hipótese $(r / q, s / q) \in \rho_{\text {erg }}(F)$, logo $0 \in \rho_{\text {erg }}\left(F^{q}-\right.$ $(r, s))$. Pela proposição anterior temos um ponto $z \in \mathbb{R}^{2}$ tal que $\rho\left(F^{q}-\right.$ $(r, s), z)=\{0\}$. Usando novamente as propriedades aritméticas de $\rho$ obtemos que $\rho(F, y)=\left\{\left(\frac{r}{q}, \frac{s}{q}\right)\right\}$ para todo $y \in \pi^{-1}(\pi(z))$.

Seja $x=\pi(z)$. Observe que $f^{q}(x)=x$, de forma que $x$ é $f$-periódico de período $q^{\prime}$ que divide $q$. Temos que $F^{q^{\prime}}(z)=z+\left(r^{\prime}, s^{\prime}\right)$ para algum $\left(r^{\prime}, s^{\prime}\right) \in \mathbb{Z}^{2}$ e calculando

$$
F^{q}(z)=z+\left(\frac{q}{q^{\prime}} r^{\prime}, \frac{q}{q^{\prime}} s^{\prime}\right)
$$

conluimos que $r=\frac{q}{q^{\prime}} r^{\prime}$ e $s=\frac{q}{q^{\prime}} s^{\prime}$. Observe, portanto, que $\frac{q}{q^{\prime}}$ divide $r, s$ e $q$ logo por hipótese $\frac{q}{q^{\prime}}=1$, ou seja, $q=q^{\prime}$.

Corolário ([MZ89], Corollary 3.11). Seja $F$ um levantamento de um homeomorfismo $f$ de $\mathbb{T}^{2}$. Então $\rho_{\text {mes }}(F) \cap \mathbb{Q}^{2}=\cup_{y \in P_{f}} \rho(F, y)$ onde $P_{f}$ é o conjunto dos pontos $y \in \mathbb{R}^{2}$ tais que $\pi(y)$ é $f$-periódico.

Demonstração. A inclusão $\rho_{m e s}(F) \cap \mathbb{Q}^{2} \subseteq \cup_{y \in P_{f}} \rho(F, y)$ é consequência da proposição anterior.

Para a outra inclusão considere $y \in P_{f}$ e obseverve que a medida de probabilidade $\mu$ com suporte em $\left\{f^{i}(\pi(y))\right\}_{i \in \mathbb{N}}$ é ergódica e que $\int \phi d \mu \in \mathbb{Q}^{2}$ pelo teorema de Birkhoff.

Aqui, a hipótese de que estamos trabalhando em $\mathbb{T}^{2}$ não pode ser dispensada. De fato, a maior parte dos resultados disponíveis hoje diz respeito ao toro de dimensão 2, e mesmo assim partes importantes da teoria continuam sem resposta (veremos mais sobre isso posteriormente). No restante desse trabalho estaremos supondo que estamos em $\mathbb{T}^{2}$, o que permite nos referirmos a $\rho(F)$ unicamente como "conjunto de rotação".

\subsection{A geometria de um conjunto de rotação}

O conjunto de rotação de um homeomorfismo do $\mathbb{T}^{2}$, homotópico à identidade, é um subconjunto compacto e convexo de $\mathbb{R}^{2}$. Desta forma ele pode ser um ponto, um segmento de reta ou possuir interior não vazio.

\section{Exemplos.}


1. Um único vetor $v$ arbitário. Como exemplo, basta considerar a translação pelo vetor $v$ em $\mathbb{R}^{2}$. É possivel construir exemplos mais interessantes, por exemplo com entropia topológica positiva (e portanto, não conjugados a uma rotação).

2. Um segmento de reta que

(a) Tenha inclinação racional e contenha um ponto de coordenadas racionais. Um exemplo é o homeomorfismo derivado do fluxo de Reeb, como definimos anteriormente, que tem $\rho(F)=[-1,1] \times$ $\{0\}$.

(b) Tenha um ponto extremo racional e todos outros pontos irracionais (esses exemplos são atribuídos a Katok). A idéia é basicamente começar com um campo de vetores de inclinação irracional em $\mathbb{R}^{2}$ e criar um ponto fixo. Os detalhes podem ser encontrados em [Beg0 7 .

3. Qualquer polígono convexo de extremos racionais [Kwa92]. O caso geral é bastante trabalhoso, mas não é difícil construir um homeomorfismo para o qual $\rho(F)=[0,1]^{2}$. Considere

$$
G(x, y)=(x+\phi(y), y), \quad H(x, y)=(x, y+\psi(x))
$$

onde $\phi, \psi: \mathbb{R} \rightarrow[0,1]$ são funções periódicas e satisfazem $\phi(t)=$ $\psi(t)=0$ se $t \in \mathbb{Z}$ e $\phi(t)=\psi(t)=1$ se $t \in \frac{1}{2}+\mathbb{Z}$. Para $F=H \circ G$ vê-se facilmente que

$$
\rho\left(F,\left(\frac{1}{2}, 0\right)\right)=(0,1), \quad \rho\left(F,\left(0, \frac{1}{2}\right)\right)=(1,0)
$$

e que

$$
\rho(F,(0,0))=(0,0), \quad \rho\left(F,\left(\frac{1}{2}, \frac{1}{2}\right)\right)=(1,1)
$$

de forma que $[0,1]^{2} \subseteq \rho(F)$. É fácil ver também que $F(x, y)-(x, y)=$ $(\phi(y), \psi(x+\phi(y)))$, de forma que $F^{n}(x, y)-(x, y) \in[0, n]^{2}$ e portanto $\rho(F) \subseteq[0,1]^{2}$. Esse exemplo foi retirado de [Beg07].

4. Em [Kwa95] é construido um exemplo em que $\rho(F)$ não é um polígono pois contém uma quantidade enumerável infinita de vértices. A construção é bastante longa e não será tratada aqui.

François Béguin propõe a seguinte questão em suas notas de aula:

Pergunta ([Beg07]). Existe um compacto convexo $K \subset \mathbb{R}^{2}$ que não é o conjunto de rotação de nenhum homeomorfismo homotópico a identidade de $\mathbb{T}^{2}$ ? 
Esse problema é mais simples se trocarmos "homeomorfismo" por "fluxo". De fato, Franks e Misiurewicz ([FM90]) mostraram que um fluxo do 2-toro cujo conjunto de rotação possui interior vazio no $\mathbb{R}^{2}$ pode ser apenas

1. Um único ponto.

2. Um segmento qualquer de uma linha determinada por zero e outro ponto de $\mathbb{Q}^{2}$.

3. Um segmento de inclinação irracional com um extremo em zero.

e que todos esses conjuntos de rotação são de fato realizados por algum fluxo em $\mathbb{T}^{2}$.

Franks e Misiurewicz conjecturaram então que, se o conjunto de rotação de um homeomorfismo $f$ está contido em uma reta ele só pode ser de um dos tipos acima. Aparentemente não houve nenhum progresso significativo na direção de provar ou desprovar esta conjectura desde que foi enunciada.

\subsection{Conjuntos de rotação e continuidade}

Um problema interessante é perguntar se $F_{n} \rightarrow F$ na topologia da convergência uniforme implica que $\rho\left(F_{n}\right) \rightarrow \rho(F)$ na topologia de Hausdorff.

É fácil ver que, em $\mathbb{T}^{2}$, a aplicação $F \mapsto \rho(F)$ é semi-continua superiormente usando que $\rho(F)=\rho_{\text {mes }}(F)$ e que o espaço das medidas é compacto na topologia $*$-fraca (mediante a identificação usual que já comentamos). Contudo, também é fácil ver com um exemplo que $F \mapsto \rho(F)$ não é semicontinua inferiormente.

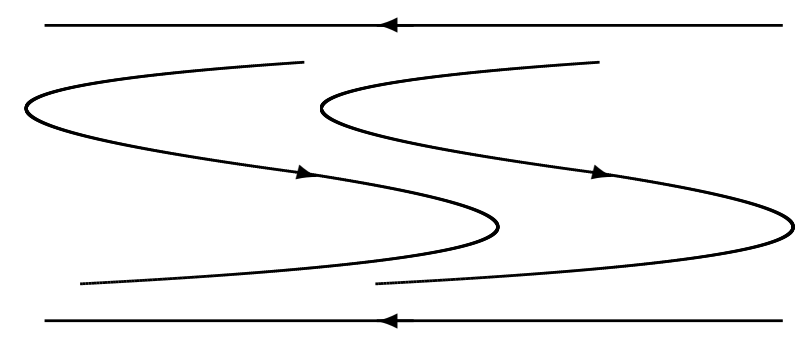

Figura 3.2: Perturbação de um fluxo de Reeb

Proposição 10 ([MZ89], Example 4.8 ; [LM91], Example 1). A função $\rho: \operatorname{Hom}_{I}\left(\mathbb{T}^{2}\right) \rightarrow \mathbb{R}^{2}$ não é semi-contínua inferiormente (aqui Hom ${ }_{I}\left(\mathbb{T}^{2}\right.$ ) denota o conjunto dos levantamentos de homeomorfismo em $\mathbb{T}^{2}$ que são homotópicos à identidade). 
Demonstração. Tomamos um campo de vetores $v$ do tipo Reeb, como definimos anteriormente, e consideramos o homeomorfismo $F$ dado pelo fluxo no tempo um. Note que $F$ é homotópico a identidade e $\rho(F)=[-1,1] \times\{0\}$.

Façamos uma perturbação $\tilde{v}$ de $v$ por "bumps" em torno de $\mathbb{R} \times\left(\frac{1}{2}+\mathbb{Z}\right)$. O homeomorfismo $\tilde{F}$ dado pelo fluxo no tempo um de $\tilde{v}$ tem conjunto de rotação igual a $\{(-1,0)\}$ (vide a figura acima).

Apesar disso segue com algum esforço e usando que $F \mapsto \rho(F)$ é semicontínua superiormente o seguinte resultado.

Proposição 11 ([LM91]). A aplicação $F \mapsto \rho(F)$ é contínua em qualquer $F$ tal que $\rho(F)$ seja um conjunto unitário.

Aqui aparece então uma distinção entre os casos em que $\rho(F)$ possui interior vazio ou não-vazio em $\mathbb{R}^{2}$.

Proposição 12 ([LM91]). A aplicação $F \mapsto \rho(F)$ é contínua em qualquer $F$ que tenha interior não-vazio.

Esse resultado é mais delicado, pois precisamos de um tipo de "estabilidade" que garanta que para todos vetores de coordenadas racionais que estejam em $\operatorname{int}(\rho(F))$ as aplicações próximas de $F$ ainda tenham pontos periódicos com esse período.

Em particular, obtemos que o conjunto dos homeomorfismos $\mathbb{Z}^{2}$-periódicos de $\mathbb{R}^{2}$ cujo conjunto de rotação tem interior não vazio é um aberto na topologia da convergência uniforme.

\subsection{Conjuntos de rotação com interior}

Exemplos como o homeomorfismo derivado do fluxo de Reeb mostram que, a princípio, apenas os pontos extremais (no sentido da análise convexa) do conjunto de rotação são realizados por pontos de $\mathbb{R}^{2}$. A seguinte proposição mostra que os pontos extremais do conjunto de rotação são, de fato, sempre realizados.

Proposição 13 ([MZ89]). Se v é um ponto extremal de $\rho(F)$ então existe $z \in \mathbb{R}^{2}$ tal que $\rho_{p}(F, z)=\{v\}$.

Demonstração. Como $\rho(F)=\operatorname{Conv}\left(\rho_{\text {erg }}(F)\right)$ todo ponto extremal de $\rho(F)$ é um ponto de $\rho_{\text {erg }}(F)$. Como argumentamos na seção 2 , o teorema de Birkhoff diz que, dado $v \in \rho_{\text {erg }}(F)$ existe $z \in \mathbb{R}^{2}$ tal que $\frac{F^{n}(z)-z}{n} \stackrel{n \rightarrow \infty}{\longrightarrow}\{v\}$, ou seja $\rho_{p}(F, z)=\{v\}$.

Pode-se mostrar que se $v$ possui ambas coordenadas racionais então $v$ é realizado por um ponto periódico. Os pontos na fronteira de $\rho(F)$ que não são extremais não precisam ser realizados (a não ser quando possuem ambas 
coordenadas racionais e estão sobre um segmento de inclinação irracional [LC05]). Observe essa propriedade nos exemplos da família que demos no item 3 no início dessa seção.

O problema geral possui uma resposta mais direta se nos restringimos aos pontos no interior de $\rho(F)$.

Proposição 14 ([Fra89]). Se $\rho(F)$ tem interior não vazio então todos os vetores de rotação com coordenadas racionais em int $(\rho(F))$ são realizados por órbitas f-periódicas.

O resultado abaixo estende a proposição anterior no caso em que um ponto em $\operatorname{int}(\rho(F))$ não possui ao menos uma das coordenadas racional.

Proposição 15 ([MZ91]). Seja $f$ um homeomorfismo de $\mathbb{T}^{2}$ e $F: \mathbb{R}^{2} \rightarrow \mathbb{R}^{2}$ um levantamento fixado. Se $v \in \operatorname{int}(\rho(F))$ então:

1. Existe um conjunto fechado $X \subseteq \mathbb{T}^{2}$, não vazio, $f$-invariante tal que $\rho_{p}(F, y)=v$ para todo $y \in \pi^{-1}(\bar{X})$.

2. Existe uma medida ergódica $\mu$, com respeito a $f$, que satisfaz

$$
\int_{\mathbb{T}^{2}}\left(F \circ \pi^{-1}-\pi^{-1}\right) d \mu=v
$$

Para finalizar essa seção comentamos mais um importante resultado.

Proposição 16 ([LM91]). Se $\rho(F)$ tem interior não vazio então $f$ tem entropia topológica estritamente positiva.

A aplicação $f=I d$ mostra que esse teorema não é verdadeiro se $\rho(F)$ tem interior vazio. É um problema interessante determinar condições adicionais sobre $f$ para garantir que, quando $\rho(F)$ tem interior vazio, $f$ tenha entropia topológica positiva.

\subsection{Conjuntos de rotação sem interior}

Como já discutimos, é fácil ver que o conjunto de rotação de uma translação $T_{v}$ por vetor $v \in \mathbb{R}^{2}$ é o próprio vetor $v$. A ação $R_{v}$ induzida por $T_{v}$ em $\mathbb{T}^{2}$ é chamada, por analogia ao caso do círculo, de uma rotação no toro.

Temos então um problema natural herdado do círculo: Quando um homeomorfismo de $\mathbb{T}^{2}$, homotópico a identidade, é conjugado com uma rotação por vetor $v$ ?

Definição 4. Dizemos que um homeomorfismo $f$, homotópico a identidade, é uma pseudo-rotação se dado um levantamento $F$ de $f$ temos que $\rho(F)=$ $\{v\}$, onde $v$ é um vetor de coordenadas irracionais. 
Consideramos apenas rotações por vetores de coordenadas irracionais pois, como vimos no caso do círculo, é necessário que todos os pontos sejam periódicos para que um homeomorfismo seja conjugado a rotação racional $R_{v}$.

O problema apresentado não é simples (ele contém no mínimo dificuldades similares ao caso do círculo, devido ao exemplo de Denjoy) e os resultados na direção de resolvê-lo são recentes. Podemos citar, por exemplo, os trabalhos de Tobias Jäger que lidam com o caso em que $v$ é um vetor de coordenadas totalmente irracionais (i.e., independentes sobre $\mathbb{Q}$ ). Precisamos da seguinte definição.

Definição 5. Dizemos que uma pseudo-rotação por vetor irracional $R_{\rho}$ tem desvio médio limitado se

$$
D(n, z):=F^{n}(z)-z-n \rho
$$

se existe $c>0$ tal que $\|D(n, z)\| \leq c$ para todo $n \in \mathbb{Z}$ e $z \in \mathbb{R}^{m}$

Proposição 17 ([Jäg08]). Seja $f$ é uma pseudo-rotação por vetor $\rho$ que preserva área e homotópica a identidade. Suponha que $f$ possui desvio médio limitado. Então vale que

1. $\rho$ é totalmente irracional se e só se $f$ é semi-conjugado a $R_{\rho}$.

2. $\rho$ é racional se, e só se, $f$ possui um ponto periódico.

Jäger mostrou posteriormente ([Jäg09]) que esse teorema não vale se $f$ não preserva área. Outro resultado interessante de Béguin, Crovisier, Le Roux e Patou, que melhorou um resultado anterior de Kwapisz no caso de difeomorfismos.

Proposição 18 ([BCRP04]). Seja $f$ uma pseudo-rotação com $\rho(F)=\{v\}$. Então $R_{v}$ pertence ao fecho da classe de conjugação de $f$.

Ao meu conhecimento, o estudo de homeomorfismos cujo conjunto de rotação é um segmento de reta é menos desenvolvido. Entre os resultados conhecidos podemos citar o resultado de Jonker e Zhang ([JZ98]), que é uma versão do teorema de Le Calvez que citamos na seção anterior (sobre pontos de coordenadas racionais contidos em segmentos de inclinação irracional) quando o conjunto de rotação não possui interior.

Ainda com respeito a existência de pontos periódicos temos também um resultado de Franks que será usado na prova de nosso teorema principal e tem um contrastre interessante com o exemplo derivado do fluxo de Reeb.

Teorema (Franks, [Fra96]). Seja $f: \mathbb{T}^{2} \rightarrow \mathbb{T}^{2}$ um homeomorfismo que preserva área e é homotópico à identidade. Seja $F: \mathbb{R}^{2} \rightarrow \mathbb{R}^{2}$ um levantamento e suponha que $\rho(F)$ é um segmento de reta. Se $v \in \rho(F)$ é um vetor com 
coordenadas racionais então existe um ponto $z \in \mathbb{R}^{2}$ tal que $\pi(z) \in \mathbb{T}^{2}$ é um ponto periódico de $f$ com $v=\rho(F, z)$. Além disso, se $v=(p / q, r / q)$, onde $p, q$ e r são relativamente primos então o período de $\pi(z)$ é $q$.

Notamos também que esse resultado admite uma versão topológica (vide [KK08]).

O resultado que apresentamos nessa tese trata do caso em que o conjunto de rotação é um segmento vertical, mas essa limitação será relaxada nos comentários finais (nosso resultado possui uma relação importante com um resultado recente de Pablo Dávalos, do qual falaremos em breve). No próximo capítulo iniciamos a motivação e explanação do problema. 


\section{Capítulo 4}

\section{A conjectura do deslocamento uniforme}

\subsection{Notação}

Chamaremos, para $i=1,2$,

$$
\begin{aligned}
& \pi_{i}: \mathbb{R}^{2} \rightarrow \mathbb{R} \\
& \quad\left(x_{1}, x_{2}\right) \mapsto x_{i}
\end{aligned}
$$

Essas duas projeções não devem ser confundidas com a projeção natural $\pi: \mathbb{R}^{2} \rightarrow \mathbb{T}^{2}$. Sempre que for conveniente preferimos a notação sufixa $(x)_{i}:=\pi_{i}(x)$.

Denotamos por $\lambda$ a medida de Lebesgue em $\mathbb{T}^{2}$.

A função |.| denota o módulo de um número real. Denotaremos por $\langle.,$. o produto interno usual do $\mathbb{R}^{2}$ e por $\|$.$\| a norma associada. \lfloor$.$\rfloor denota a$ função chão (que associa a $x \in \mathbb{R}$ o maior inteiro menor que $x$ ).

Seja $R$ um subconjunto dos números reais $\mathbb{R}$. Dadas duas funções $f, g$ : $R \rightarrow \mathbb{R}$ diremos que $f \in o(g(x))$ se e somente se para todo $M \in \mathbb{R}^{+}$existe $x_{0} \in R$ tal que

$$
|f(x)| \leq M|g(x)| \text { para todo } \quad x>x_{0}
$$

também diremos que $f \in O(g(x))$ se e somente se existe $M \in \mathbb{R}^{+}$e $x_{0} \in R$ tais que

$$
|f(x)| \leq M|g(x)| \text { para todo } \quad x>x_{0}
$$

Note que $o(g(x)) \subseteq O(g(x))$.

\subsection{Motivação da conjectura}

Como notamos anteriormente decorre de que $f$ é homotópico a identidade que $F(x)-x=F(x+(p, q))-(x+(p, q))$ para todo $(p, q) \in \mathbb{Z}^{2}$, de forma 
que dado $x \in \mathbb{T}^{2}$ a função

$$
\left(F^{n} \circ \pi^{-1}-\pi^{-1}\right): \mathbb{T}^{2} \rightarrow \mathbb{R}^{2}
$$

está bem definida. Suponha agora que $\rho(F)=\{0\} \times[a, b]$ com $a \leq b$. É evidente que, dado $x \in \mathbb{T}^{2}$, para todo $y \in \pi^{-1}(x)$,

$$
\left(F^{n}(y)-y\right)_{1} \in o(n)
$$

e em particular $\left(F^{n}(y)-y\right)_{1} \in O(n)$, de forma que o crescimento desta função em termos de $n$ é no máximo linear. A priori a hipótese sobre a forma do conjunto de rotação permite que $\left(F^{n}(y)-y\right)_{1} \in o(\ln (n))$, pois $\lim _{n \rightarrow \infty} \frac{\ln (n)}{n}=0$, de forma que $\left(F^{n}(y)-y\right)_{1}$ não precisa tender a zero e portanto não precisa pertencer a $o(1)$. No entanto, ainda podemos nos perguntar se é possível que $\left(F^{n}(y)-y\right)_{1} \in O(1)$ (ou seja, se há deslocamento sublinear).

Conjectura (do deslocamento uniforme). Seja $f: \mathbb{T}^{2} \rightarrow \mathbb{T}^{2}$ um homeomorfismo homotópico a identidade e $F: \mathbb{R}^{2} \rightarrow \mathbb{R}^{2}$ um levantamento fixado de forma que $\rho(F)=\{0\} \times[a, b] \subseteq \mathbb{R}^{2}$, onde $a<b$.

Nessas condiçôes existe $M>0$ tal que $\left|\left(F^{n}(x)-x\right)_{1}\right|<M$ para todo $n \in \mathbb{Z}, x \in \mathbb{R}$.

Podemos ainda motivar esta conjectura da seguinte maneira: Como vimos no capítulo anterior, a geometria do conjunto $\rho(F)$ pode implicar na existência de pontos do plano que se movem assintóticamente em diferentes direções pela ação de $F$. Em particular vemos que quando $\rho(F)$ possui interior não vazio e quando $\rho(F)$ é um segmento de rela não vertical podemos afirmar que existe $x \in \mathbb{R}^{2}$ tal que $\left|\left(F^{n}(x)-x\right)_{1}\right|$ é ilimitado quando $n \rightarrow \infty$. Será verdade então que quando $\rho(F)$ é um segmento de reta vertical (eventualmente degenerado) passando por $(0,0)$ temos que $\left|\left(F^{n}(x)-x\right)_{1}\right|$ é uniformemente limitado?

Curiosamente, a conjectura do deslocamento uniforme é falsa no caso em que $\rho(F)=\{(0,0)\}$. Isto foi provado recentemente por Koropecki e Tal (vide [KT12a]), portanto vemos que a hipótese de que $a<b$ é indispensável. Mesmo assim ainda não sabemos se essa conjectura é válida, a não ser sobre as hipóteses adicionais que incluiremos a seguir e devido no caso do seguinte resultado.

Proposição 19 ([Dáv11]). Seja $f$ um homeomorfismo de $\mathbb{T}^{2}$ homotópico a identidade e suponha que existe levantamente $F: \mathbb{R}^{2} \rightarrow \mathbb{R}^{2}$ tal que $\rho(F)=$ $\{0\} \times[a, b]$ com $a<0<b$. Então ou todo ponto de coordenadas racionais em $\rho(F)$ é realizado ou para todo $x \in \mathbb{R}^{2}$ existe $M>0$ tal que $\left|\left(F^{n}(x)-x\right)_{1}\right|<$ $M$ para todo $n \in \mathbb{N}$.

Note em particular que se $F$ preserva área o teorema de Franks garante que todo ponto de coordenadas racionais em $\rho(F)$ é realizado, logo o resultado acima não contempla a conjectura quando a área é preservada. 


\subsection{Nosso resultado}

O que faremos a seguir é demonstrar a seguinte versão parcial da conjectura do deslocamento uniforme.

Teorema. Seja $f: \mathbb{T}^{2} \rightarrow \mathbb{T}^{2}$ um homeomorfismo homotópico a identidade $e$ $F: \mathbb{R}^{2} \rightarrow \mathbb{R}^{2}$ um levantamento fixado de forma que $\rho(F)=\{0\} \times[a, b] \subseteq \mathbb{R}^{2}$, onde $a<b$.

Suponhamos que $f$ é ergódica com respeito a medida de Lebesgue $\lambda$. Suponhamos também que o vetor de rotação médio com respeito a esta medida

$$
\rho_{\lambda}(F):=\int_{x \in \mathbb{T}^{2}}\left(F \circ \pi^{-1}(x)-\pi^{-1}(x)\right) d \lambda=(0, \alpha)
$$

para algum $\alpha$ irracional. Então existe $M>0$ tal que $\left|\left(F^{n}(x)-x\right)_{1}\right|<M$ para todo $n \in \mathbb{Z}, x \in \mathbb{R}$.

Note que, como $f$ é homotópico a identidade, é suficiente mostrar o desejado para $x \in[0,1]$. Dado $m \in \mathbb{Z}$ a função $F^{m}-I d$ é contínua, portanto $\left(F^{m}-I d\right)[0,1]$ é limitado. Como para $m \geq 0$ temos que $\rho\left(F^{m}\right)=m \rho(F)$ e supomos que $\rho(F)$ é um segmento de reta não-degenerado podemos assumir, fazendo se necessário uma troca de levantamento, que $(0,0),(0,1),(0,-1) \in$ $\rho(F)$.

O teorema pode ser generalizado para alguns outros conjuntos de rotação que são segmentos de reta não degenerados. Isso pode ser feito de maneira simples que portanto deixamos para tratar ao final do texto. Também discutiremos posteriormente esse resultado dentro de uma teoria mais geral, que busca entender alguns homeomorfismos homotópicos a identidade do 2-toro como aplicações do anel e por sua vez aplicar os resultados disponíveis nesse contexto.

A conjectura do deslocamento uniforme também pode ser estudada no caso em que $f$ é homotópica a um Dehn Twist. Ela foi provada por AddasZanata, Garcia e Tal ([GTAZ11]), mas nesse caso a teoria difere do que discutimos no capítulo anterior e por isso não a discutiremos aqui.

A ferramenta que usaremos em nosso caso é estudar os conjuntos $\omega\left(B_{0}\right)$ e $\omega\left(B_{\pi}\right)$ que serão descritos no capítulo seis. Estes conjuntos, que estão sendo estudados por Addas-Zanata e Tal há alguns anos, inicialmente em conexão com a conjectura de Boyland, descrevem alguns conjuntos contínuos (isto é, conexos e completos) na compactificação do plano por um ponto com boas propriedades dinâmicas. A presença destas estruturas permite obtermos muita informação sobre a dinâmica discreta determinada por $f \mathrm{e}$ pode ser informalmente comparada a resultados da teoria de Brouwer que descrevemos no próximo capítulo. 


\subsection{O papel da ergodicidade}

A hipótese de ergodicidade com respeito a medida de Lebsegue em associação com as outras hipóteses do teorema que queremos provar implica, entre outras coisas, que para $\lambda$-quase todo ponto $p \in \mathbb{T}^{2}$ existe uma sequência $n_{j} \stackrel{j \rightarrow \infty}{\longrightarrow} \infty$ tal que

$$
\left(F^{n_{j}}(y)-y\right)_{1} \stackrel{j \rightarrow \infty}{\longrightarrow} 0
$$

para todo $y \in \pi^{-1}(p)$. Para provar essa afirmação usaremos o lema abaixo que é uma consequência do teorema de Atkinson (vide [Atk76]).

Lema 3 (Atkinson). Seja $M$ uma variedade compacta e $f: M \rightarrow M$ contínua. Seja $\mu$ uma medida boreliana, de probabilidade, ergódica. Seja $g: M \rightarrow \mathbb{R}$ continua e satisfazendo

$$
\int_{M} g d \mu=0
$$

para $\mu$-qtp existe sequência $n_{k} \stackrel{k \rightarrow \infty}{\longrightarrow} \infty$ tal que

$$
f^{n_{k}}(x) \stackrel{k \rightarrow \infty}{\longrightarrow} x
$$

$e$

$$
\sum_{i=n_{0}}^{n_{k}-1} g\left(f^{i}(x)\right) \stackrel{k \rightarrow \infty}{\longrightarrow} 0
$$

Definindo $g:=\left(F \circ \pi^{-1}-\pi^{-1}\right)_{1}$ e pondo $\mu=\lambda$ sabemos que $\lambda\left(\pi\left(B_{\varepsilon}(x)\right)\right)>$ 0 para todo $\varepsilon>0$ e $x \in \mathbb{R}^{2}$. Como $\lambda$ é ergódica o teorema de Birkhoff garante que, para $\lambda$-quase todo $p \in \pi\left(B_{\varepsilon}(x)\right)$, temos

$$
\frac{1}{n} \sum_{i=n_{0}}^{n-1} g\left(f^{i}(p)\right) \stackrel{k \rightarrow \infty}{\longrightarrow} \int_{\mathbb{T}^{2}} g d \lambda
$$

Então se assumirmos que $\rho(F)$ está contido em $\{0\} \times \mathbb{R}$ e como para todo $p \in \mathbb{T}^{2}$ e $y \in \pi^{-1}(p)$ temos que

$$
\sum_{i=0}^{j} g\left(f^{i}(p)\right)=\sum_{i=0}^{j}\left(F^{i+1}(y)-F^{i}(y)\right)_{1}=\left(F^{j+1}(y)-y\right)_{1}
$$

concluímos que $\int_{\mathbb{T}^{2}}\left(F \circ \pi^{-1}-\pi^{-1}\right)_{1} d \lambda=0$. Podemos aplicar então o lema de Atkinson para obter uma sequência $n_{j} \stackrel{j \rightarrow \infty}{\longrightarrow}-\infty$ e $p \in \pi\left(B_{\varepsilon}(x)\right)$ tal que para $\lambda$-quase todo $y \in \pi^{-1}(p)$ temos que $f^{n_{j}}(p) \stackrel{j \rightarrow \infty}{\longrightarrow} p$ e $\left(F^{n_{j}+1}(y)-\right.$ $y)_{1} \stackrel{j \rightarrow \infty}{\longrightarrow} 0$ como desejado. 
Os homeomorfismos ergódicos, por sua vez, são um $G_{\delta}$ (i.e., intersecção enumerável de abertos) denso na topologia da convergência uniforme dentre os homeomorfismos do toro que preservam a medida de Lebesgue no toro (esse resultado é conhecido como teorema de Oxtoby-Ulam; uma boa referência para o assunto é [AP00]).

A hipótese de que o vetor de rotação médio possui segunda coordenada irracional é um pouco mais técnica e será usada em momentos distintos da prova do teorema. Basicamente ela garante que a dinâmica é suficientemente complicada, a princípio além do que é garantido pela ergodicidade e o teorema de Franks. 
26CAPÍTULO 4. A CONJECTURA DO DESLOCAMENTO UNIFORME 


\section{Capítulo 5}

\section{Os conjuntos $B_{0}, B_{\pi}$ e $\omega\left(B_{0}\right), \omega\left(B_{\pi}\right)$}

\subsection{Definições; $B_{0}, B_{\pi} \neq \emptyset$}

Fixemos a notação $e_{0}=(1,0)$ e $e_{\pi}=(-1,0)$. Definimos

$$
V_{0}^{+}:=\left\{x \in \mathbb{R}^{2} \mid\left\langle x, e_{0}\right\rangle \geq 0\right\}=\left\{x \in \mathbb{R}^{2} \mid(x)_{1} \geq 0\right\}
$$

e

$$
V_{\pi}^{+}:=\left\{x \in \mathbb{R}^{2} \mid\left\langle x, e_{\pi}\right\rangle \geq 0\right\}=\left\{x \in \mathbb{R}^{2} \mid(x)_{1} \leq 0\right\}
$$

Considere $\mathbb{R}^{2} \cup\{\infty\} \sim S^{2}$ a compactificação por um ponto de $\mathbb{R}^{2}$ e $\widehat{F}$ o homeomorfismo induzido por $F$ em $S^{2}$ que fixa o infinito. Os conjuntos $\widehat{V_{0}^{+}}:=V_{0}^{+} \cup\{\infty\}$ e $\widehat{V_{\pi}^{+}}:=V_{\pi}^{+} \cup\{\infty\}$ correspondem a $V_{0}^{+}$e $V_{\pi}^{+}$, respectivamente, em $S^{2}$.

Seja $\widehat{B_{0}}$ a componente conexa de

$$
\bigcap_{n \leq 0} \widehat{F}^{n} \widehat{\left(V_{0}^{+}\right)}
$$

que contém o infinito. Seja também $\widehat{B_{\pi}}$ como a componente conexa de

$$
\bigcap_{n \leq 0} \widehat{F}^{n}\left(\widehat{V_{\pi}^{+}}\right)
$$

que contém o infinito.

Podemos definir então os conjuntos $B_{0}$ e $B_{\pi}$ em $\mathbb{R}^{2}$ que correspondem, respectivamente, aos conjuntos $\widehat{B_{0}}$ e $\widehat{B_{\pi}}$ de $S^{2}$. Vamos mostrar que estes conjuntos não são vazios, sob as hipóteses de nosso teorema, com o lema que é consequência dos resultados a seguir. 
Proposição 20. Seja $f: \mathbb{T}^{2} \rightarrow \mathbb{T}^{2}$ um homeomorfismo homotópico a identidade e seja $F$ um levantamento fixado com $\rho(F)=\{0\} \times[a, b]$. Suponha que $f$ é ergódico com respeito a medida de Lebesgue no toro. Se existe $M \in \mathbb{R}$, $n(M) \in \mathbb{Z}$ e $x \in \mathbb{R}^{2}$ tal que $\left(F^{n(M)}(x)-x\right)_{1}>M$ então existe $z \in \mathbb{R}^{2} e$ $m(M) \in \mathbb{Z}$ tal que $\left(F^{m(M)}(z)-z\right)_{1}<-M+1$.

Demonstração. Seja $M>0, n(M) \in \mathbb{Z}$ e $x_{M} \in \mathbb{R}^{2}$ tais que $\left(F^{n(M)}\left(x_{M}\right)-\right.$ $\left.x_{M}\right)_{1}>M$. Por continuidade dado $\varepsilon>0$ existe $\delta_{M}>0$ tal que para todo $y \in B_{\delta_{M}}\left(x_{M}\right)$ temos

$$
M-\varepsilon<\left(F^{n(M)}(y)-y\right)_{1}<M+\varepsilon
$$

Aplique o lema de Atkinson, como fizemos na seção anterior, para $g:=$ $\left(F \circ \pi^{-1}-\pi^{-1}\right)_{1}$. Podemos fazer isso pois $\lambda\left(\pi\left(B_{\delta_{M}}\left(x_{M}\right)\right)\right)>0$ e como para todo $p \in \mathbb{T}^{2}$ e $y \in \pi^{-1}(p)$ temos que

$$
\sum_{i=0}^{j} g\left(f^{i}(p)\right)=\sum_{i=0}^{j}\left(F^{i+1}(y)-F^{i}(y)\right)_{1}=\left(F^{j+1}(y)-y\right)_{1}
$$

temos, como já vimos, pelo teorema de Birkhoff que $\int_{\mathbb{T}^{2}}\left(F \circ \pi^{-1}-\pi^{-1}\right)_{1} d \lambda=$ 0 . Obtemos assim uma sequência $n_{j} \stackrel{j \rightarrow \infty}{\longrightarrow} \infty$ e $p \in \pi\left(B_{\varepsilon}(x)\right)$ tal que para todo $y \in \pi^{-1}(p)$ temos que $f^{n_{j}}(p) \stackrel{j \rightarrow \infty}{\longrightarrow} p$ e

$$
\left(F^{n_{j}+1}(y)-y\right)_{1} \stackrel{j \rightarrow \infty}{\longrightarrow} 0
$$

Temos também que

$$
\left(F^{n_{j}+1}(y)-F^{n(M)}(y)\right)_{1}=\left(F^{n_{j}+1}(y)-y\right)_{1}+\left(y-F^{n(M)}(y)\right)_{1}
$$

logo por (5.1) e (5.2) temos que $\left(F^{n_{j}}(y)-F^{n(M)}(y)\right)_{1} \stackrel{j \rightarrow \infty}{\longrightarrow}-M$. Se defi$\operatorname{nirmos} z:=F^{n(M)}(y)$ então

$$
\left(F^{n_{j}+1}(y)-F^{n(M)}(y)\right)_{1}=\left(F^{n_{j}+1-n(M)}(z)-z\right)_{1}
$$

de forma que

$$
\left(F^{n_{j}+1-n(M)}(z)-z\right)_{1} \stackrel{j \rightarrow \infty}{\longrightarrow}-M
$$

como desejamos. A demonstração para $M<0$ é análoga.

Proposição 21. Seja $f: \mathbb{T}^{2} \rightarrow \mathbb{T}^{2}$ um homeomorfismo homotópico a identidade e $F$ um levantamento fixado de $f \operatorname{com} \rho(F)=\{0\} \times[a, b]$. Suponha que $f$ é ergódico com respeito a medida de Lebesgue no toro. Se F possui um ponto fixo então $B_{0}$ e $B_{\pi}$ não são vazios. 
Demonstração. Seja $y \in \mathbb{R}^{2}$ um ponto fixo. Como $F(x+(i, j))=F(x)+$ $(i, j)$ podemos assumir que $0 \leq(y)_{1} \leq 1$. Seja $L:=\left\{x \in \mathbb{R}^{2} \mid(x)_{1}=(y)_{1}\right\}$, i.e., $L$ é a reta vertical passando por todos os pontos (fixos) da forma $y+(0, i)$ para $i \in \mathbb{Z}$. Usando a proposição anterior podemos mostrar que $B_{0} \neq \emptyset$ dividindo em dois casos:

1. Suponha que existe $M>0$ inteiro tal que para todo $x \in \mathbb{R}^{2}$ e $m \in \mathbb{Z}$ temos que $\left|\left(F^{m}(x)-x\right)_{1}\right| \leq M$. Em particular temos que $\mid\left(F^{m}(L)-\right.$ $y)_{1} \mid \leq M$. Observe que $L$ divide $\mathbb{R}^{2}$ em duas componentes conexas

$$
\left(V_{\pi}^{+}+\left((y)_{1}, 0\right)\right) \backslash L \quad \text { e } \quad\left(V_{0}^{+}+\left((y)_{1}, 0\right)\right) \backslash L
$$

Como $F^{m}(L)$ é ilimitada na vertical para todo $m \leq 0$, podemos definir uma orientação "para cima"e dizer quando um conjunto está a esquerda ou a direita de $F^{m}(L)$. Note que também temos pontos fixos em $\left(V_{0}^{+}+\left((y)_{1}, 0\right)\right) \backslash L$. Como $F^{-1}$ é contínua preserva o número de componentes conexas, portanto estes pontos fixos garantem que a imagem da componente conexa que está a direita de $L$ também está a direita de $F^{-1}(L)$.

Como $\left|\left(F^{m}(L)-y\right)_{1}\right| \leq M$ temos para todo $n \in \mathbb{Z}$

$$
F^{n}(L+(M, 0)) \subseteq V_{0}^{+}
$$

Observe também que $V_{0}^{+}+\left((y)_{1}+M, 0\right)$ está a direita de $L+(M, 0)$, de forma que $F^{n}\left(V_{0}+\left(M+(y)_{1}, 0\right)\right)$ está a direita de $F^{n}(L+(M, 0))$ e portanto, para todo $n \in \mathbb{Z}$, temos que

$$
V_{0}^{+}+\left(M+(y)_{1}, 0\right) \subseteq F^{m}\left(V_{0}^{+}\right)
$$

logo o conjunto ilimitado $V_{0}^{+}+\left(M+(y)_{1}, 0\right)$ está em $B_{0}$.

2. Pela proposição anterior podemos supor que para todo $M>0$ existe $x_{M} \in \mathbb{R}^{2}$ e $n(M) \in \mathbb{Z}$ tal que $\left(F^{n(M)}\left(x_{M}\right)-x_{M}\right)_{1}>M$. Note que, como $F^{n(M)}-I d$ é invariante pela ação de $\mathbb{Z}^{2}$ podemos tomar $x_{M}$ a esquerda de $L \operatorname{com} F^{n(M)}\left(x_{M}\right)$ a direita de $L$.

Em particular dado $M>0$ existe $n(M) \in \mathbb{Z}$ o primeiro inteiro tal que $F^{m}(L) \cap\left(V_{0}^{+}+(M, 0)\right) \neq \emptyset$. Seja $M_{k}$ uma sequência ilimitada de inteiros e $\gamma_{k}$ uma curva conexa ilimitada para a direita e que começa em $F^{m\left(M_{k}\right)}(L) \cap\left(V_{0}^{+}+\left(M_{k}, 0\right)\right)$.

Temos então que $\delta_{k}:=F^{-m\left(M_{k}\right)}\left(\gamma_{k}\right)$ está a direita de $L$. Como $F$ é homotópica a identidade posso assumir, s.p.g., que o ponto inicial de cada curva $\delta_{k}$ está em $\left[(y)_{1},(y)_{1}+1\right] \times[0,1]$. Também podemos ver que $F^{i}\left(\delta_{k}\right) \subseteq V_{0}^{+}$para $n\left(M_{k}\right) \leq i \leq 0$ utilizando que os pontos fixos garantem que a imagem da componente conexa que está a direira de $L$ também está a direita de $F(L)$. 
Olhe então para as curvas $\widehat{\delta_{k}} \in \mathbb{S}^{2}$ que correspondem as curvas $\delta_{k} \in \mathbb{R}^{2}$. As curvas $\widehat{\delta_{k}} \in \mathbb{S}^{2}$ formam uma sequência de conjuntos compactos conexos que portanto possui (na topologia de Hausdorff) uma subsequência convergindo para um conjunto fechado conexo $\widehat{\Gamma}$, que está a direita de $L$ pois $\delta_{k} \rightarrow \Gamma$ e $F\left(\delta_{k}\right)$ está a direita de $L$. Note que $\widehat{\Gamma} \neq\{\infty\}$ pois a sequência dos pontos iniciais das curvas $\delta_{k}$ converge para um ponto de $\left.\left[(y)_{1},(y)_{1}+1\right] \times[0,1]\right)$ Desta forma o conjunto correspondente $\Gamma \subset \mathbb{R}^{2}$ mostra que $B_{0} \neq \emptyset$.

A prova de que $B_{\pi} \neq \emptyset$ pode ser obtida analogamente.

No teorema acima podemos argumentar por um raciocínio semelhante sem as hipóteses $\rho(F)=\{0\} \times[a, b]$ e de ergodicidade para obter que ao menos um dos conjuntos $B_{0}, B_{\pi}$ é não-vazio (pois ou as imagens de $L$ são uniformemente limitadas em módulo - caso 1 - ou são ilimitadas em ao menos um dos dois lados - caso 2).

Lema 4. Seja F um levantamento fixado de um homeomorfismo homotópico a identidade que é ergódico com repeito a medida de Lebsegue. Se $\rho(F)=$ $\{0\} \times[a, b] \subseteq \mathbb{R}^{2}$, onde $a \leq 0 \leq b$ então $B_{0}$ e $B_{\pi}$ não são vazios.

Demonstração. Se $a<b$, como $(0,0) \in \rho(F)$, pelo teorema de Franks existe $x \in \mathbb{T}^{2}$ tal que

$$
F(y)-y=(0,0)
$$

para todo $y \in \pi^{-1}(x)$. Para o caso $a=b=0$ temos que $(0,0)$ é um ponto extremal e a existência do ponto fixo pode ser vista como consequência de outro resultado de Franks que já citamos (proposição 8, página 12). Basta aplicar então a proposição anterior.

Em tempo, conhecemos outras condições sobre as quais $B_{0}$ e $B_{\pi}$ são não vazios. Veja por exemplo [GTAZ11] e [Tal12].

Precisaremos também das seguintes caracterizações dos conjuntos $B_{0} \mathrm{e}$ $B_{\pi}$ :

Lema 5. $B_{0}$ é a união de todas os conjuntos conexos fechados, ilimitados $C$ de $\mathbb{R}^{2}$ que satisfazem

$$
\left(F^{n}(x)\right)_{1} \geq 0 \forall n \in \mathbb{N}^{+}
$$

para todo $x \in C$. Analogamente, $B_{\pi}$ é a união de todos os conjuntos conexos fechados, ilimitados $D$ de $\mathbb{R}^{2}$ que satisfazem

$$
\left(F^{n}(x)\right)_{1} \leq 0 \forall n \in \mathbb{N}^{+}
$$

para todo $x \in D$. 
Demonstração. Provaremos apenas a primeira afirmação, pois a segunda é análoga. Por definição $\widehat{B_{0}}$ é conexo e fechado. Claramente $B_{0}$ não precisa ser conexo, pois retiramos o ponto no infinito, mas cada uma de suas componentes conexas precisa ser conexa e fechada, além de ilimitada. Como $B_{0}$ é a união de suas componentes conexas, basta ver que a órbita futura de seus pontos permanece em $V_{0}^{+}$para sempre. Mas isso é consequência direta da forma que definimos $\widehat{F}$ e $\widehat{V_{0}^{+}}$.

Observe também que, como $\widehat{V_{0}^{+}}, \widehat{V_{\pi}^{+}}$são fechados e $\widehat{F}$ é homeomorfismo, $\widehat{B_{0}}, \widehat{B_{\pi}}$ são fechados. Assim, $B_{0}, B_{\pi}$ são fechados (o que não segue do lema anterior).

Definimos então o $\omega$-limite de $B_{0}$, que será denotado por

$$
\omega\left(B_{0}\right):=\bigcap_{i=1}^{\infty} \overline{\bigcup_{j=i}^{\infty} F^{j}\left(B_{0}\right)}=\bigcap_{i=1}^{\infty} F^{i}\left(B_{0}\right)
$$

(pois $F$ é homeomorfismo, $B_{0}$ é fechado e $F^{i+1}\left(B_{0}\right) \subseteq F^{i}\left(B_{0}\right)$ para $i \geq 0{ }^{1}$ ). Analogamente

$$
\omega\left(B_{\pi}\right):=\bigcap_{i=1}^{\infty} F^{i}\left(B_{\pi}\right)
$$

Argumentando como no lema anterior podemos ver que $\omega\left(B_{0}\right)$ e $\omega\left(B_{\pi}\right)$ são fechados e todas suas componentes conexas são ilimitadas. Veja também que $\omega\left(B_{0}\right) \subseteq B_{0}\left(\right.$ pois $\left.F\left(B_{0}\right) \subseteq B_{0}\right)$ e que $\omega\left(B_{\pi}\right) \subseteq B_{\pi}$.

É fácil ver que $F\left(\omega\left(B_{0}\right)\right)=\omega\left(B_{0}\right)$. Usando que $F$ é um homeomorfismo $F^{-1}\left(\omega\left(B_{0}\right)\right)=\omega\left(B_{0}\right)$. Vemos por indução que $F^{i}\left(\omega\left(B_{0}\right)\right)=\omega\left(B_{0}\right)$ para todo $i \in \mathbb{Z}$. Nesse caso diremos que $\omega\left(B_{0}\right)$ e $\omega\left(B_{\pi}\right)$ são totalmente invariantes.

Também precisaremos mais a frente do seguinte resultado:

Proposição 22. Os conjuntos $B_{0}^{C}, B_{\pi}^{C}, \omega\left(B_{0}\right)^{C}$ e $\omega\left(B_{\pi}\right)^{C}$ possuem, cada um, uma única componente conexa.

Demonstração. Vamos provar que $B_{0}^{C}$ possui uma única componente conexa. O restante da proposição pode ser provado de forma análoga.

É claro que $B_{0} \subseteq V_{0}^{+}$, de forma que podemos considerar $\Omega$ a componente conexa de $B_{0}^{C}$ que contém $\mathbb{R}^{2} \backslash V_{0}^{+}$. Como $B_{0}$ é $F$-invariante temos que $F\left(B_{0}^{C}\right) \supseteq B_{0}^{C}$, de forma que toda componente conexa de $F\left(B_{0}^{C}\right)$ contém uma componente conexa de $B_{0}^{C}$ (lembre que um homeomorfismo preserva o número de componentes conexas). Além disso $F(\Omega) \cap \Omega \neq \emptyset$ e vale que $\Omega \subseteq F(\Omega)$. Em particular, $\Omega^{C}$ é $F$-invariante.

Seja $z \in \Omega^{C}$ e $\Gamma$ a componente conexa de $\Omega^{C}$ que contém $z$. Como $\Omega^{C}$ é invariante temos que $F^{i}(\Gamma) \cap \Omega=\emptyset$ para todo $i \in \mathbb{N}$. Como $\left(V_{0}^{+}\right)^{C} \subseteq \Omega$

\footnotetext{
${ }^{1}$ Isto não foi provado, mas é trivial. Basta supor que $F\left(B_{0}\right) \nsubseteq B_{0}$ e ver o que acontece com o respectivo conjunto em $S^{2}$.
} 
temos que $\Gamma$ é um conjunto fechado, conexo cuja órbita futura não sai de $V_{0}^{+}$.

Além disso, como $\partial\left(\Omega^{C}\right) \subseteq B_{0}$, temos que $\Gamma \cap B_{0} \neq \emptyset$. Seja $z \in \Gamma \cap B_{0}$ e $\Theta$ a componente conexa de $B_{0}$ que contém $z$. Sabemos que $\Theta$ é ilimitado e como $\Theta \cap \Gamma \neq \emptyset$ então $\Theta \subseteq \Gamma$. Portanto $\Gamma$ é ilimitado, fechado e sua órbita futura não sai de $V_{0}^{+}$. Concluímos que $\Gamma \subseteq B_{0}$; isso mostra que $\Omega^{C}=B_{0}$, e tem portanto uma única componente conexa.

\section{$5.2 \omega\left(B_{0}\right), \omega\left(B_{\pi}\right) \neq \emptyset$}

Veremos nessa seção que, sob nossas hipóteses, podemos assumir que $\omega\left(B_{0}\right)$ e $\omega\left(B_{\pi}\right)$ são ambos não vazios.

Proposição 23. Se $(p, q) \in \mathbb{Z}^{2}$ com $p \geq 0$ então $B_{0}+(p, q) \subseteq B_{0}$. Além disso, $B_{0}+(0, q)=B_{0}$.

Demonstração. Seja $\Gamma$ uma componente conexa de $B_{0}$. Claramente $\Gamma+$ $(p, q)$ é conexo, fechado, ilimitado e tal que $\left(F^{i}(y)\right)_{1} \geq 0$ para todo $y \in$ $\Gamma+(p, q)\left(\right.$ pois $\left.\left(F^{i}(y)\right)_{1} \geq p \geq 0\right)$.

Assim, se $x \in \Gamma \subseteq B_{0}$ então $(x+(p, q)) \in(\Gamma+(p, q)) \subseteq B_{0}$. Por fim, note que se $p=0$ então segue a igualdade do lema 5 .

Podemos facilmente ver que vale um resultado análogo para $B_{\pi}$ quando $p \leq 0$. Em tempo, a proposição seguinte é semelhante e será usada mais a frente.

Proposição 24. Se $(p, q) \in \mathbb{Z}^{2}$ com $p \geq 0$ então $\omega\left(B_{0}\right)+(p, q) \subseteq \omega\left(B_{0}\right)$. Se $(p, q) \in \mathbb{Z}^{2}$ com $p \leq 0$ então $\omega\left(B_{\pi}\right)+(p, q) \subseteq \omega\left(B_{\pi}\right)$. Além disso, se $p=0$ então vale a igualdade em ambos os casos.

Demonstração. Como $\omega\left(B_{0}\right)=\bigcap_{i=1}^{\infty} F^{i}\left(B_{0}\right)$ temos

$$
\omega\left(B_{0}\right)+(p, q)=\left(\bigcap_{i=1}^{\infty} F^{i}\left(B_{0}\right)\right)+(p, q)=\bigcap_{i=1}^{\infty} F^{i}\left(B_{0}+(p, q)\right)
$$

Usando que $F$ é homeomorfismo temos da proposição anterior que $\omega\left(B_{0}\right)+$ $(p, q) \subseteq \omega\left(B_{0}\right)$ e que vale a igualdade caso $p=0$. O restante do pedido é obtido analogamente.

Lema 6. Se $\omega\left(B_{0}\right)=\emptyset$ então existe $r \in \mathbb{R}_{*}^{+}$tal que

$$
\liminf _{n \rightarrow \infty} \frac{\left(F^{n}(x)\right)_{1}}{n} \geq r
$$

para todo $x \in B_{0}$. 
Demonstração. Afirmo que existe $n \in \mathbb{N}^{+}$tal que $F^{i}\left(B_{0}\right) \subseteq V_{0}^{+}+(1,0)$ para todo $i>n$. De fato, caso contrário existe uma sequência $i_{j} \rightarrow \infty$ tal que, para todo $j$, existe um ponto $x_{j} \in V_{0}^{+}$tal que $F^{i_{j}}\left(x_{j}\right) \in V_{0}^{+} \backslash\left(V_{0}^{+}+\right.$ $(1,0))$ e portanto $0 \leq\left(F^{i_{j}}\left(x_{j}\right)\right)_{1}<1$.

Os inteiros $q_{j}=\left\lfloor\left(F^{i_{j}}\left(x_{j}\right)\right)_{2}\right\rfloor$ são tais que $F^{i_{j}}\left(x_{j}\right)-\left(0, q_{j}\right) \in[0,1]^{2}$. Como $x_{j} \in V_{0}^{+} \subseteq B_{0}$ e $B_{0}-\left(0, q_{j}\right)=B_{0}$ temos que $x_{j}-\left(0, q_{j}\right) \in B_{0}$. Mas por compacidade o conjunto $\left\{F^{i_{j}}\left(x_{j}-\left(0, q_{j}\right)\right)\right\}_{j \in \mathbb{N}}$ possui uma subsequência convergindo para um ponto $w \in \omega\left(B_{0}\right)$ : contradição, pois $\omega\left(B_{0}\right)=\emptyset$.

Afirmo agora que $F^{n}\left(B_{0}\right) \subseteq B_{0}+(1,0)$. Seja $x \in B_{0}$. Pela afirmação anterior, $F^{i}(x) \in V_{0}^{+}+(1,0)$, ou ainda, $F^{i}(x)-(1,0) \in V_{0}^{+}$para $i>n \mathrm{e}$ desta forma $F^{i}(x)-(1,0) \in B_{0}$ o que prova a afirmação. Veja por indução que, para todo $k \in \mathbb{N}^{+}$temos que

$$
F^{n k}\left(B_{0}\right) \subseteq B_{0}+(k, 0) \subseteq V_{0}^{+}+(k, 0)
$$

de forma que para todo $x \in B_{0}$

$$
\liminf _{n \rightarrow \infty} \frac{\left(F^{n}(x)\right)_{1}}{n} \geq \frac{k}{n k}=\frac{1}{n}
$$

Corolário. Se $\omega\left(B_{0}\right)=\emptyset$ então existe $v \in \rho(F) \operatorname{com}(v)_{1}>0$.

Demonstração. Pelo lema anterior e pela proposição 21 existe $x \in \mathbb{R}^{2}$, $r \in \mathbb{R}$ e $N \in \mathbb{N}$ suficientemente grande tal que $\frac{\left(F^{n}(x)\right)_{1}}{n} \geq r>0$ para todo $n>N$. Desta forma

$$
\left(\rho_{n}(F, x)\right)_{1}=\frac{\left(F^{n}(x)-x\right)_{1}}{n} \geq r-\frac{(x)_{1}}{n} \stackrel{n \rightarrow \infty}{\longrightarrow} r>0
$$

Seja $\left\{x_{i}\right\}_{i} \in \mathbb{N}$ uma sequência em $B_{0}$ contém infinitos pontos podemos considerar

$\rho(F)$ é compacto podemos extrair uma subsequência de $\left\{\frac{F^{n}(x)-x}{n}\right\}_{n>N}$ que converge para algum $v \in \rho(F) \operatorname{com}(v)_{1}>0$.

De forma análoga podemos ver que

Corolário. Se $\omega\left(B_{\pi}\right)=\emptyset$ então existe $v \in \rho(F) \operatorname{com}(v)_{1}<0$.

Concluímos que, para um homeomorfismo com conjunto de rotação que coincide com um segmento de reta vertical, é suficiente estudar o caso em que $\omega\left(B_{0}\right)$ e $\omega\left(B_{\pi}\right)$ são ambos não vazios.

\subsection{Estratégia da prova}

A partir do próximo capítulo assumiremos que o teorema que desejamos provar não vale. Vamos então dividir a prova do teorema nos casos $\pi\left(\omega\left(B_{0}\right)\right) \cap$ 
$\pi\left(\omega\left(B_{\pi}\right)\right)=\emptyset$ ou $\pi\left(\omega\left(B_{0}\right)\right) \cap \pi\left(\omega\left(B_{\pi}\right)\right) \neq \emptyset$ e mostrar que ambos não podem acontecer: esse absurdo produz a prova do teorema.

Falemos brevemente desses dois estágios da prova. Supomos primeiramente que $\pi\left(\omega\left(B_{0}\right)\right) \cap \pi\left(\omega\left(B_{\pi}\right)\right) \neq \emptyset$ e olhamos para o conjunto aberto $A:=$ $\left(\omega\left(B_{0}\right) \cup\left(\omega\left(B_{\pi}\right)+(p, q)\right)\right)^{C}$. Se $(p, q)$ é tal que $\omega\left(B_{0}\right) \cap\left(\omega\left(B_{\pi}\right)+(p, q) \neq \emptyset\right.$ podemos mostrar que $A$ possui infinitas componentes conexas distintas.

O lema de Atkinson é usado então para observar que uma componente conexa $O$ de $A$ precisa eventualmente interceptar (e portanto coincidir) com a componente conexa $O+(0, i)$ (que provaremos ser distinta de $O$ se $i \neq 0$ ). Isso leva eventualmente a conclusão que um conjunto aberto possui vetor de deslocamento médio com segunda coordenada racional, o que contradiz uma de nossas hipóteses e prova que o caso $\pi\left(\omega\left(B_{0}\right)\right) \cap \pi\left(\omega\left(B_{\pi}\right)\right) \neq \emptyset$ não pode ocorrer.

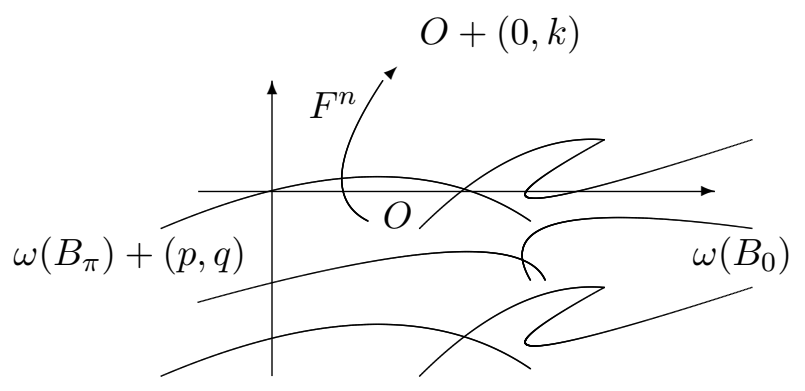

Figura 5.1: O conjunto $A:=\left(\omega\left(B_{0}\right) \cup\left(\omega\left(B_{\pi}\right)+(p, q)\right)\right)^{C}$ deveria possuir infinitas componentes conexas que sobem com velocidade média racional, uma contradição.

Assumiremos então que $\pi\left(\omega\left(B_{0}\right)\right) \cap \pi\left(\omega\left(B_{\pi}\right)\right)=\emptyset$ e mostramos que, sob nossas hipóteses, esses conjuntos são densos em $\mathbb{T}^{2}$. Iremos então escolher um ponto não-fixo $x \in \omega\left(B_{0}\right)$ e transportar uma componente conexa $\Theta$ de $\omega\left(B_{\pi}\right)$ de forma que a distância entre ela e a componente conexa $\Gamma$ que passa por $x$ seja suficientemente pequena.

Ligamos essas duas componentes conexas por uma reta $v$ : Mostramos então que o conjunto $\Gamma \cup v \cup \Theta$ divide o plano em duas componentes conexas que chamaremos $\Omega_{1}$ e $\Omega_{2}$. 


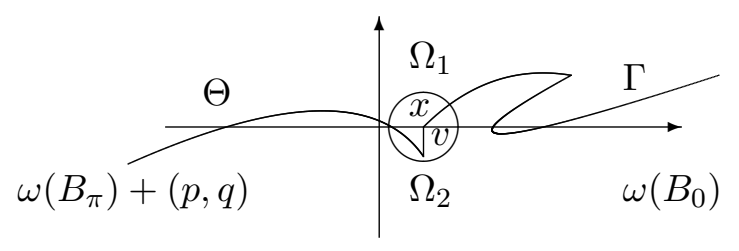

Figura 5.2: Escolhemos $(p, q) \in \mathbb{Z}^{2}$ tais que uma componente conexa $\Theta$ fique suficientemente próxima da componente conexa $\Gamma$ que passa por $x \in \omega\left(B_{0}\right)$. Ligamos essas componentes por um segmento $v$.

A escolha de $x \in \omega\left(B_{0}\right)$ e $v$ como acima garantirá que ou $F^{k}\left(\overline{\Omega_{1}}\right) \subseteq \overline{\Omega_{1}}$ ou $F^{k}\left(\overline{\Omega_{2}}\right) \subseteq \overline{\Omega_{2}}$ para todo $k \in \mathbb{N}$. Mas isto não pode acontecer, pois o teorema de Franks dá, sob nossas hipóteses, pontos periódicos que no plano sempre sobem ou sempre descem e podemos provar que esses pontos estão tanto em $\Omega_{1}$ quanto em $\Omega_{2}$. Isso é novamente uma contradição que leva a conclusão desejada.

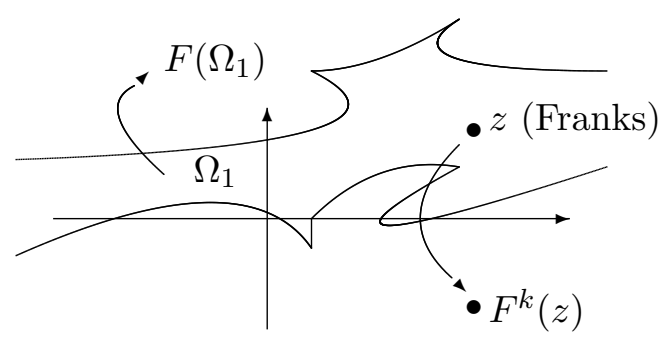

Figura 5.3: Caso em que $F\left(\overline{\Omega_{1}}\right) \subseteq \overline{\Omega_{1}}$. No entanto o teorema de Franks fornece um ponto de $\Omega_{1}$ que deve "descer" para $\Omega_{2}$, uma contradição. 


\section{Capítulo 6}

\section{Prova do teorema}

\subsection{Consequências da negação do teorema}

A prova do teorema será por contradição, de forma que por todo este capítulo estaremos admitindo que o teorema não vale, a menos de quando notado diferente. Isso permite que simplifiquemos significativamente as hipóteses dos resultados a seguir.

Definimos o conjunto

$$
A:=\left(\omega\left(B_{0}\right) \cup\left(\omega\left(B_{\pi}\right)+(p, q)\right)\right)^{C}
$$

O conjunto $A$ depende a priori de $p$ e $q$ mas, como não vale o teorema 1 , podemos mostrar que $A$ nunca é vazio.

Proposição 25. Seja $M \in \mathbb{R}^{+}$. Existe $x \in \mathbb{R}^{2}$ e $n(x) \in \mathbb{Z}$ tais que

$$
(x)_{1}<-M \quad \text { e } \quad\left(F^{n(x)}(x)\right)_{1} \geq M
$$

Em particular, $A \neq \emptyset$ para todo $(p, q) \in \mathbb{Z}^{2}$.

Demonstração. Como estamos supondo que não vale o teorema sabemos que existe $n(x)$ tal que $\left|\left(F^{n(x)}(x)-x\right)_{1}\right| \geq 2 M+1$. Vamos supor que $\left(F^{n(x)}(x)-x\right)_{1}>0$, de forma que $\left|\left(F^{n(x)}(x)-x\right)_{1}\right|=\left(F^{n(x)}(x)-x\right)_{1}$.

Por definição $0 \leq(x)_{1}-\left\lfloor(x)_{1}\right\rfloor<1$. O ponto $x_{0}:=x-\left(\left\lfloor(x)_{1}\right\rfloor+M+1,0\right)$ satisfaz

$$
-M-1 \leq\left(x_{0}\right)_{1}<-M
$$

Como $\left(x_{0}\right)_{1}<-M$ então $x_{0} \notin V_{0}^{+}$e claramente $x_{0} \notin \omega\left(B_{0}\right)$.

Mas, como $\left(\left\lfloor(x)_{1}\right\rfloor+M+1,0\right) \in \mathbb{Z}^{2}$, obtemos que

$$
\left(F^{n(x)}\left(x_{0}\right)-x_{0}\right)_{1}=\left(F^{n(x)}(x)-x\right)_{1} \geq 2 M+1
$$

o que por sua vez implica que

$$
\left(F^{n(x)}\left(x_{0}\right)\right)_{1} \geq 2 M+1+\left(x_{0}\right)_{1} \geq 2 M+1-M-1=M
$$


Portanto $x_{0}$ também não está em $\left(\omega\left(B_{\pi}\right)+(M-1, q)\right)$ já que uma de suas iteradas tem primeira coordenada maior ou igual que $M$. Tomando em particular $M=p+1$ concluímos que $x \in A$.

Precisamos considerar o caso $\left(F^{n(x)}(x)-x\right)_{1}<0$. Nesse caso faça $y:=$ $F^{n(x)}(x)$ e observe que $F^{-n(x)}(y):=x$, de forma que $\left(y-F^{-n(x)}(y)\right)_{1}<0$ ou seja $\left(F^{-n(x)}(y)-y\right)_{1}>0$. Recaímos portanto no caso anterior, o que completa a prova.

Proposição 26. Todas as componentes conexas de $\omega\left(B_{0}\right)$ e $\omega\left(B_{\pi}\right)$ são ilimitadas na horizontal.

Demonstração. Vamos mostrar que todas as componentes conexas de $\omega\left(B_{0}\right)$ e $\omega\left(B_{\pi}\right)$ não podem ser ilimitadas somente na vertical. Em particular, como já sabemos que estas são ilimitadas, concluiremos que todas as componentes conexas de $\omega\left(B_{0}\right)$ e $\omega\left(B_{\pi}\right)$ são ilimitadas na horizontal.

Suponha que alguma componente conexa $C$ de $\omega\left(B_{0}\right)$ é ilimitada na vertical mas com comprimento horizontal finito. Então, para $i \in \mathbb{Z}, C+$ $(0, i)$ também é uma componente conexa de $\omega\left(B_{0}\right)$ ilimitada na vertical mas limitada na horizontal.

Claramente $\cup_{i=-\infty}^{+\infty}(C+(0, i))$ separa os conjuntos $R:=\left\{x \in \mathbb{R}^{2} \mid(x)_{1} \geq\right.$ $M\}$ e $L:=\left\{x \in \mathbb{R}^{2} \mid(x)_{1} \leq-M\right\}$ para $M \geq \sup _{x \in C}\left|(x)_{1}\right|$. Sabemos que $\omega\left(B_{0}\right)^{C}$ possui uma única componente conexa, de forma que ou $L$ ou $R$ está completamente contido $\omega\left(B_{0}\right)^{C}$.

Suponha que $L$ está completamente contido em $\omega\left(B_{0}\right)^{C}$. Pela proposição anterior algum ponto de $\omega\left(B_{0}\right)^{C}$ tem uma de suas iteradas com primeira coordenada maior ou igual que $M+1$ e portanto pertence a $R$. Mas isso é uma contradição, pois $\omega\left(B_{0}\right)^{C}$ é totalmente invariante e $L \cap R=\emptyset^{1}$.

O caso em que $R$ está completamente contido em $\omega\left(B_{0}\right)^{C}$ é análogo, fazendo como antes: tome $y:=F^{n(x)}(x)$ e observe que $(y)_{1} \geq M$ e que $\left(F^{-n(x)(y)}\right)_{1}=(x)_{1} \leq-M$. Isso também leva a uma contradição, pois vimos que $\omega\left(B_{0}\right)^{C}$ é totalmente invariante e $L \cap R=\emptyset$.

A prova para as componentes conexas de $\omega\left(B_{\pi}\right)$ segue analogamente.

\subsection{O caso $\pi\left(\omega\left(B_{0}\right)\right) \cap \pi\left(\omega\left(B_{\pi}\right)\right) \neq \emptyset$ não pode ocorrer}

Nessa seção mostraremos que, se não vale o teorema, então $\pi\left(\omega\left(B_{0}\right)\right) \cap$ $\pi\left(\omega\left(B_{\pi}\right)\right)=\emptyset$.

Se $\pi\left(\omega\left(B_{0}\right)\right) \cap \pi\left(\omega\left(B_{\pi}\right)\right) \neq \emptyset$ então existem $x \in \mathbb{R}^{2}$ e $\left(p_{1}, q_{1}\right),\left(p_{2}, q_{2}\right) \in$ $\mathbb{Z}^{2}$ tais que

$$
\left(\omega\left(B_{0}\right)+\left(p_{1}, q_{1}\right)\right) \cap\left(\omega\left(B_{\pi}\right)+\left(p_{2}, q_{2}\right)\right) \neq \emptyset
$$

Para simplificar a notação faça $(p, q)=\left(p_{2}-p_{1}, q_{2}-q_{1}\right)$, logo existe $x \in \mathbb{R}^{2}$ tal que

$$
x \in \omega\left(B_{0}\right) \cap\left(\omega\left(B_{\pi}\right)+(p, q)\right)
$$

\footnotetext{
${ }^{1}$ Pois sabemos que $\omega\left(B_{0}\right)$ é totalmente invariante
} 
Precisaremos das seguintes proposições

Proposição 27. Se O é uma componente conexa de

$$
A=\left(\omega\left(B_{0}\right) \cup\left(\omega\left(B_{\pi}\right)+(p, q)\right)\right)^{C}
$$

então $O+(0, i)$ é uma componente conexa de A para todo $i \in \mathbb{Z}$.

Demonstração. Pela proposição 24 temos que $A^{C}+(0, i)=A^{C}$ para todo $i \in \mathbb{Z}$. Isso implica que $A+(0, i)=A$ para todo $i \in \mathbb{Z}$, o que prova o desejado pois $A$ é a união de suas componentes conexas.

Proposição 28. Seja $O$ uma componente conexa de $A$ e seja $x \in O$. Então $(x+(0, i)) \notin O$ para todo $i \in \mathbb{Z}^{*}$. Em particular, $O$ e $O+(0, i)$ são componentes conexas distintas de $A$ para todo $i \in \mathbb{Z}^{*}$.

Demonstração. Defina para $i \in \mathbb{Z}$

$$
y(i):=x+i(0,1)
$$

Observe que $y(i) \in A$ para todo $i \in \mathbb{Z}$. Vamos mostrar que todos os pontos $y(i)$ estão em diferentes componentes conexas de $A$.

Suponha que não. Em particular, existem $i_{1}<i_{2}$ tais que $y\left(i_{1}\right)$ e $y\left(i_{2}\right)$ estão na mesma componente conexa de $A$. Seja $\alpha$ uma curva limitada na horizontal que liga $y\left(i_{1}\right)$ e $y\left(i_{2}\right)$ e não intercepta $A^{C}$. As curvas $\alpha+(0, i)$ também não interceptam $A^{C}$, para $i \in \mathbb{Z}$.

Temos então que a curva $\beta:=\cup_{k \in \mathbb{Z}}\left(\alpha+k\left(0, i_{2}-i_{1}\right)\right)$ é conexa, ilimitada na vertical, limitada na horizontal e não intercepta $A^{C}$. Note que $\beta$ separa os conjuntos $R:=\left\{x \in \mathbb{R}^{2} \mid(x)_{1}>M\right\}$ e $L:=\left\{x \in \mathbb{R}^{2} \mid(x)_{1}<-M\right\}$ para $\operatorname{algum} M>0$.

Pela proposição 26, as componentes conexas de $\omega\left(B_{0}\right)$ e $\omega\left(B_{\pi}\right)$ são ilimitadas na horizontal. Seja $z \in \omega\left(B_{0}\right) \cap\left(\omega\left(B_{\pi}\right)+(p, q)\right)$, seja $\Gamma_{1}$ a componente conexa de $\omega\left(B_{0}\right)$ e $\Gamma_{2}$ a componente conexa de $\omega\left(B_{\pi}\right)+(p, q)$ que o contém. Como $\omega\left(B_{0}\right) \subseteq V_{0}^{+}, \omega\left(B_{\pi}\right) \subseteq V_{\pi}^{+}$concluímos que $\Gamma:=\Gamma_{1} \cup \Gamma_{2}$ é conexa, ilimitada nas duas direções horizontais e $\Gamma \cap A=\emptyset$. Contradição, pois $\Gamma \cap \beta \neq \emptyset$.

Lembre que, como $\lambda$ é ergódica, temos para $\lambda$-qtp $x \in \mathbb{T}^{2}$ que

$$
\lim _{n \rightarrow \infty} \frac{F^{n} \circ \pi^{-1}(x)-\pi^{-1}(x)}{n}=\int_{x \in \mathbb{T}^{2}}\left(F^{n} \circ \pi^{-1}(x)-\pi^{-1}(x)\right) d \lambda=(0, \alpha)
$$

ou seja, para quase todo ponto (com respeito a medida de Lebesgue $\lambda$ em $\left.\mathbb{T}^{2}\right)$ o vetor de rotação pontual existe e é da forma $(0, \alpha)$ para algum $\alpha$ irracional. Com essa observação e as duas proposições anteriores podemos mostrar que

Proposição 29. Se não vale o teorema então $\pi\left(\omega\left(B_{0}\right)\right) \cap \pi\left(\omega\left(B_{\pi}\right)\right)=\emptyset$. 
Demonstração. Vamos supor por absurdo que $\omega\left(B_{0}\right) \cap\left(\omega\left(B_{\pi}\right)+(p, q)\right) \neq \emptyset$ para algum $(p, q) \in \mathbb{Z}^{2}$. Pela proposição 25 temos que existe $x \in A$.

Como $A$ é aberto se $O$ é a componente conexa de $A$ que contém $x$ então existe $\varepsilon>0$ tal que $B_{\varepsilon}(x) \subseteq O$. Observe que, para todo $\varepsilon>0$ temos $\lambda\left(\pi\left(B_{\varepsilon}(x)\right)\right)>0$. Como fizemos na proposição 20 seja $g:=\left(F \circ \pi^{-1}-\pi^{-1}\right)_{1}$. Então, pelo lema de Atkinson, existe sequência $n_{j} \stackrel{j \rightarrow \infty}{\longrightarrow} \infty$ e $p \in \pi\left(B_{\varepsilon}(x)\right)$ tal que para $\lambda$-quase todo $y \in \pi^{-1}(p)$ temos que $f^{n_{j}}(p) \stackrel{j \rightarrow \infty}{\longrightarrow} p$ e

$$
\left(F^{n_{j}}(y)-y\right)_{1} \stackrel{j \rightarrow \infty}{\longrightarrow} 0
$$

Podemos assumir que existe $\rho_{p}(F, y)$, pois o vetor de rotação existe para quase todo ponto. Note também que para algum $k_{j} \in \mathbb{Z}$ temos $F^{n_{j}}(y) \in$ $\left(B_{\varepsilon}(x)+\left(0, k_{j}\right)\right)$. Mas $F^{n_{j}}$ é contínua e $A$ é $F$-invariante, logo $F$ leva componente conexa de $A$ em componente conexa de $A$. Pela proposição anterior

$$
F^{n_{j}}(O)=O+\left(0, k_{j}\right)
$$

Por indução vemos facilmente para todo $s \in \mathbb{N}$ que

$$
F^{s n_{j}}(O)=O+s\left(0, k_{j}\right)
$$

Podemos assumir também que existem $j_{1}, j_{2} \in \mathbb{N}$ tais que $\frac{k_{j_{1}}}{n_{j_{1}}} \neq \frac{k_{j_{2}}}{n_{j_{2}}}$. Para ver isso, suponha que $\frac{k_{j_{1}}}{n_{j_{1}}}=\frac{k_{j_{m}}}{n_{j_{m}}}$ para todo $m \in \mathbb{N}$. Temos que

$$
k_{j_{m}}-\varepsilon \leq\left(F^{n_{j_{m}}}(y)-y\right)_{2} \leq k_{j_{m}}+\varepsilon
$$

dividindo por $n_{j_{m}}$ podemos tomar o limite quando $m \rightarrow \infty$ para ver (como existe $\left.\rho_{p}(F, y)\right)$ que

$$
\alpha=\lim _{m \rightarrow \infty} \frac{k_{j_{m}}}{n_{j_{m}}}=\frac{k_{j_{1}}}{n_{j_{1}}}
$$

um absurdo, pois $\alpha$ é irracional.

Usando (6.1) para $n_{j_{2}}$ e $n_{j_{1}}$ temos que

$$
O+n_{j_{1}}\left(0, k_{j_{2}}\right)=F^{n_{j_{1}} n_{j_{2}}}(O)=F^{n_{j_{2}} n_{j_{1}}}(O)=O+n_{j_{2}}\left(0, k_{j_{1}}\right)
$$

Mas isso é um absurdo pois pelo lema anterior, como $\frac{k_{j_{1}}}{n_{j_{1}}} \neq \frac{k_{j_{2}}}{n_{j_{2}}}$, temos que $O+n_{j_{1}}\left(0, k_{j_{2}}\right) \neq O+n_{j_{2}}\left(0, k_{j_{1}}\right)$.

\subsection{A distância entre $\pi\left(\omega\left(B_{0}\right)\right)$ e $\pi\left(\omega\left(B_{\pi}\right)\right)$}

Já provamos que se não vale o teorema então $\pi\left(\omega\left(B_{0}\right)\right) \cap \pi\left(\omega\left(B_{\pi}\right)\right)=\emptyset$. Vamos provar agora que se $\pi\left(\omega\left(B_{0}\right)\right) \cap \pi\left(\omega\left(B_{\pi}\right)\right)=\emptyset$ então, sob nossas hipóteses, a distância entre $\pi\left(\omega\left(B_{0}\right)\right)$ e $\pi\left(\omega\left(B_{\pi}\right)\right)$ é zero. Na verdade, provaremos ainda mais: 
Proposição 30. Vale que $\overline{\pi\left(\omega\left(B_{0}\right)\right)}=\mathbb{T}^{2}=\overline{\pi\left(\omega\left(B_{\pi}\right)\right)}$.

Começamos a prova deste resultado com uma proposição.

Proposição 31. Para todo ponto $z \in \mathbb{T}^{2}$ e para todo $\varepsilon>0$, existe uma curva $\gamma \subset \mathbb{R}^{2}$ ilimitada na vertical, limitada na horizontal e tal que para todo $y \in \gamma$ existe $n(y) \leq 0$ tal que $\pi\left(F^{n(y)}(y)\right) \in B_{\varepsilon}(z)$.

Demonstração. Como fizemos na demonstração do resultado anterior, podemos usar o lema de Atkinson para encontrar, para cada $x \in \mathbb{R}^{2}$, um $y \in B_{\varepsilon}(x), n_{j} \in \mathbb{N}^{*}$ e $k_{j} \in \mathbb{Z}$ para os quais $F^{n_{j}}(y) \in B_{\varepsilon}(x)+\left(0, k_{j}\right)$. Também como antes podemos assumir que existem $j_{1}, j_{2} \in \mathbb{N}$ tais que $\frac{k_{j_{1}}}{n_{j_{1}}} \neq \frac{k_{j_{2}}}{n_{j_{2}}}$.

Defina $O_{0}=B_{\varepsilon}(x)$, onde $x \in \pi^{-1}(z)$, e para todo $n \geq 1$ defina

$$
O_{n}=F^{n_{j_{1}}}\left(O_{n-1}\right) \cup\left(B_{\varepsilon}(x)+n\left(0, k_{j_{1}}\right)\right)
$$

Observe que $F^{n_{j_{1}}}\left(O_{0}\right) \cap\left(B_{\varepsilon}(x)+\left(0, k_{j_{1}}\right)\right) \neq \emptyset$, de forma que $O_{1}$ é conexo. Como $B_{\varepsilon}(x)+\left(0, k_{j_{1}}\right) \subseteq O_{1}$ e $F$ comuta com as translações inteiras temos que $F^{n_{j_{1}}}\left(O_{1}\right) \cap\left(B_{\varepsilon}(x)+2\left(0, k_{j_{1}}\right)\right) \neq \emptyset$, de forma que $O_{2}$ é conexo. Vemos facilmente por indução que $O_{n}$ é conexo para todo $n \in \mathbb{N}$.

Definimos analogamente $V_{0}=B_{\varepsilon}(x)$ e para todo $n \geq 1$ defina

$$
V_{n}=F^{n_{j_{2}}}\left(V_{n-1}\right) \cup\left(B_{\varepsilon}(x)+n\left(0, k_{j_{2}}\right)\right)
$$

Claramente, $V_{n}$ é conexo para todo $n \in \mathbb{N}$.

Além disso queremos ver que $O_{n_{j_{2}}} \cap V_{n_{j_{1}}} \neq \emptyset$. Para isso observe que segue das definições

$$
O_{n_{j_{2}}} \supseteq F^{n_{j_{1}}}\left(O_{n_{j_{2}}-1}\right) \supseteq F^{2 n_{j_{1}}}\left(O_{n_{j_{2}}-2}\right) \supseteq \ldots \supseteq F^{n_{j_{2}} n_{j_{1}}}\left(B_{\varepsilon}(x)\right)
$$

e

$$
V_{n_{j_{1}}} \supseteq F^{n_{j_{2}}}\left(V_{n_{j_{1}}-1}\right) \supseteq F^{2 n_{j_{2}}}\left(V_{n_{j_{1}}-2}\right) \supseteq \ldots \supseteq F^{n_{j_{1}} n_{j_{2}}}\left(B_{\varepsilon}(x)\right)
$$

Concluímos que $O_{n_{j_{2}}} \cup V_{n_{j_{1}}}$ é conexo, de forma que contém uma curva $\delta$ limitada na horizontal ligando $x+\left(0, n_{j_{2}} k_{j_{1}}\right)$ com $x+\left(0, n_{j_{1}} k_{j_{2}}\right)$. Como $k:=\left|n_{j_{2}} k_{j_{1}}-n_{j_{1}} k_{j_{2}}\right| \neq 0$ então $\gamma=\cup_{i \in \mathbb{Z}}(\delta+i(0, k))$ satisfaz ao pedido.

Proposição 32. $\overline{\pi\left(\omega\left(B_{0}\right)\right)}=\mathbb{T}^{2}$.

Demonstração. Suponha que existe $\pi(x) \in \mathbb{T}^{2}$ e $\varepsilon>0$ tal que, para todo $\pi(w) \in \pi\left(\omega\left(B_{0}\right)\right)$, temos que $\pi(w) \notin B_{\varepsilon}(\pi(x))$.

Seja $\Gamma$ a componente conexa de $\omega\left(B_{0}\right)$ que passa por $w \in \pi^{-1}(\pi(w))$. Como não vale o teorema, $\Gamma$ é ilimitada na horizontal.

Pela proposição anterior, dado $\pi(x) \in \mathbb{T}^{2}$ e $\varepsilon>0$ existe curva $\gamma$ tal que para todo $y \in \gamma$ existe $n(y) \leq 0$ tal que $\pi\left(F^{n(y)}(y)\right) \in B_{\varepsilon}(\pi(x))$. Além disso $\gamma$ é limitada na horizontal e ilimitada na vertical, de forma que para algum $x \in \pi^{-1}(\pi(x))$ temos que $\Gamma \cap \gamma \neq \emptyset$.

Mas se $z \in \Gamma \cap \gamma$ então $z \in \omega\left(B_{0}\right)$ e existe $n(z) \leq 0$ tal que $\pi\left(F^{n(z)}(z)\right) \in$ $B_{\varepsilon}\left(\pi(x)\right.$ ). Em particular, $F^{n(z)}(z) \in \omega\left(B_{0}\right)$ (pois $\omega\left(B_{0}\right)$ é totalmente invariante) e $\pi\left(F^{n(z)}(z)\right) \in B_{\varepsilon}(\pi(x))$ o que contradiz a hipótese de absurdo.

Mostra-se analogamente que $\overline{\pi\left(\omega\left(B_{\pi}\right)\right)}=\mathbb{T}^{2}$. 


\subsection{O caso $\pi\left(\omega\left(B_{0}\right)\right) \cap \pi\left(\omega\left(B_{\pi}\right)\right)=\emptyset$ não pode ocorrer}

Vamos provar a seguir que se $\pi\left(\omega\left(B_{0}\right)\right) \cap \pi\left(\omega\left(B_{\pi}\right)\right)=\emptyset$ então vale o teorema. Precisaremos do seguinte lema

Lema 7. Suponha que $\pi\left(\omega\left(B_{0}\right)\right) \neq \emptyset$. Então o conjunto

$$
A^{C}=\omega\left(B_{0}\right) \cup\left(\omega\left(B_{\pi}\right)+(p, q)\right)
$$

possui ao menos um ponto que não é fixo.

Demonstração. Basta mostrar que $\omega\left(B_{0}\right)$ contém um ponto que não é fixo. Mas já sabemos que $\pi\left(\omega\left(B_{0}\right)\right)$ é denso em $\mathbb{T}^{2}$, de forma que se $\pi\left(\omega\left(B_{0}\right)\right) \subseteq$ Fix $(f)$ teríamos que $f=I d$. Nesse caso vemos facilmente que $\rho(F)$ é um único ponto, uma contradição.

Seja $x \in \omega\left(B_{0}\right)$ e suponha que $x$ não é fixo. Por continuidade isso diz que existe uma $\varepsilon$-vizinhança de $x$ tal que $F\left(B_{\varepsilon}(x)\right) \cap B_{\varepsilon}(x)=\emptyset$. Como, sob nossas hipóteses, $\overline{\pi\left(\omega\left(B_{\pi}\right)\right)}=\mathbb{T}^{2}$ existe $y \in \mathbb{R}^{2}$ tal que $\pi(y) \in B_{\varepsilon}(\pi(x))$ e $\pi(y) \in \pi\left(\omega\left(B_{0}\right)\right)$, ou seja, existem $p_{1}, q_{1} \in \mathbb{Z}$ tais que $y \in \omega\left(B_{\pi}\right)+\left(p_{1}, q_{1}\right)$.

Existem também $p_{2}, q_{2} \in \mathbb{Z}$ tais que $\left\|\left(y+\left(p_{2}, q_{2}\right)\right)-x\right\|<\varepsilon$ (pois $\pi(y) \in$ $\left.B_{\varepsilon}(\pi(x))\right)$ e $y+\left(p_{2}, q_{2}\right) \in \omega\left(B_{\pi}\right)+\left(p_{1}+p_{2}, q_{1}+q_{2}\right)$.

Como $\pi\left(\omega\left(B_{0}\right)\right) \cap \pi\left(\omega\left(B_{\pi}\right)\right)=\emptyset$ a distância entre os conjuntos compactos $\omega\left(B_{0}\right) \cap \overline{B_{\varepsilon}(x)}$ e $\left(\omega\left(B_{\pi}\right)+\left(p_{1}+p_{2}, q_{1}+q_{2}\right)\right) \cap \overline{B_{\varepsilon}(x)}$ é um número estritamente positivo $d$. Note também que $d \leq \varepsilon$.

Chamaremos de $v$ o segmento aberto de reta de comprimento $d$ ligando um ponto $x_{1} \in \omega\left(B_{0}\right) \cap \overline{B_{\varepsilon}(x)}$ a um ponto $y_{1} \in\left(\omega\left(B_{\pi}\right)+\left(p_{1}+p_{2}, q_{1}+q_{2}\right)\right)$. Observe em particular que, como $F\left(B_{\varepsilon(x)}\right) \cap B_{\varepsilon(x)}=\emptyset$, temos que $F(v) \cap v=$ $\emptyset$. Seja $\Gamma$ a componente conexa de $\omega\left(B_{0}\right)$ que contém $x_{1}$ e $\Theta$ a componente conexa de $\omega\left(B_{\pi}\right)+(p, q)$ que contém $y_{1}$. Citamos o seguinte resultado.

Proposição 33 (Vide [New39]). Sejam $K_{1}, K_{2}$ conjuntos compactos conexos de $\mathbb{R}^{2}$ tais que $K_{1} \cap K_{2}$ é conexo. Se $x, y$ estão na mesma componente conexa de $K_{1}^{C}$ e também na mesma componente conexa de $K_{2}^{C}$ então $x$ e y estão na mesma componente conexa de $\left(K_{1} \cup K_{2}\right)^{C}$.

Temos então a seguinte proposição.

Proposição 34. Suponha que $\pi\left(\omega\left(B_{0}\right)\right) \cap \pi\left(\omega\left(B_{\pi}\right)\right)=\emptyset$. Então o conjunto

$$
A=\left(\omega\left(B_{0}\right) \cup\left(\omega\left(B_{\pi}\right)+(p, q)\right)\right)^{C}
$$

possui uma única componente conexa.

Demonstração. Suponha que existem $x$ e $y$ pertencentes a diferentes componentes conexas de $A$. Como $\omega\left(B_{0}\right)^{C}$ e $\omega\left(B_{\pi}\right)^{C}$ são fechados e possuem, cada um, uma única componente conexa existem curvas conexas de comprimento finito $\gamma_{1}$ e $\gamma_{2}$ ligando $x$ a $y$ e contidas, respectivamente, em $\omega\left(B_{0}\right)^{C}$ e $\left(\omega\left(B_{\pi}\right)+(p, q)\right)^{C}$. Seja $M>\max \left\{\left\|\gamma_{1}\right\|,\left\|\gamma_{2}\right\|\right\}$. 
Tomemos

$$
K_{1}=\left(\omega\left(B_{0}\right) \cap B_{M}[0]\right) \cup \partial B_{M}[0]
$$

e

$$
K_{2}=\left(\left(\omega\left(B_{\pi}\right)+(p, q)\right) \cap B_{M}[0]\right) \cup \partial B_{M}[0]
$$

Os conjuntos $K_{1}$ e $K_{2}$ são fechados e limitados na métrica euclideana, portanto compactos. Além disso, $K_{1}$ e $K_{2}$ são conexos, já que todas as componentes conexas de $\omega\left(B_{0}\right)$ e $\omega\left(B_{\pi}\right)$ são ilimitadas e assim precisam cruzar $\partial B_{M}[0]$. Como $\omega\left(B_{0}\right) \cap\left(\omega\left(B_{\pi}\right)+(p, q)\right)=\emptyset$ vemos que $K_{1} \cap K_{2}=\partial B_{M}[0]$, que é conexo.

Notamos agora que quaisquer dois pontos $x, y$ fora de $K_{1}$ e $K_{2}$ estão na mesma componente conexa de $K_{1}^{C}$ e na mesma componente conexa de $K_{2}^{C}$ (pois $\omega\left(B_{0}\right)^{C}$ e $\omega\left(B_{\pi}\right)^{C}$ possuem, cada um, uma única componente conexa). Pela proposição anterior temos então que $x$ e $y$ estão na mesma componente conexa de $\left(K_{1} \cup K_{2}\right)^{C}$.

Desta forma, existe uma curva conexa ligando $x$ a $y$ sem tocar $K_{1} \cup K_{2}$ e portanto que em particular não sai de $B_{M}[0]$ (pois para isso precisaria tocar $\left.\partial B_{M}[0]\right)$. Como essa curva também não intercepta $\omega\left(B_{0}\right) \cap B_{M}[0]$ e $\left(\omega\left(B_{\pi}\right)+(p, q)\right) \cap B_{M}[0]$, vemos que $x$ e $y$ pertencem a mesma componete conexa de $A$, uma contradição.

Com isso obtemos que

Lema 8. O conjunto $(\Gamma \cup \Theta)^{C}$ possui uma única componente conexa. O conjunto $(\Gamma \cup v \cup \Theta)^{C}$ possui exatamente duas componentes conexas.

Demonstração. A primeira afirmação é consequência da proposição anterior. Vejamos então que $(\Gamma \cup v \cup \Theta)^{C}$ possui no máximo duas componentes conexas. Pela escolha de $v$ temos que, se $v_{m}$ é o ponto médio do segmento $v$, temos que $\Gamma \cap \overline{B_{\frac{d}{2}}\left(v_{m}\right)}=\{x\}$ e $\Theta \cap \overline{B_{\frac{d}{2}}\left(v_{m}\right)}=\{y\}$, onde $d$ é o comprimento de $v$. Cada uma das metades de $\overline{B_{\frac{d}{2}}\left(v_{m}\right)} \backslash v$ está entá contida em uma única componente conexa de $(\Gamma \cup v \cup \Theta)^{C}$. Chamamos essas metades de $E$ e $D$.

Suponha agora que $a, b$ pertencem a diferentes componentes conexas de de $(\Gamma \cup v \cup \Theta)^{C}$. Vejamos que qualquer $c \in(\Gamma \cup v \cup \Theta)^{C}$ pertence ou a componente conexa de $a$ ou a componente conexa de $b$. De fato, caso contrário, como $(\Gamma \cup \Theta)^{C}$ possui uma única componente conexa existem curvas simples, parametrizadas por $[0,1]$, denotadas $l_{1}$ e $l_{2}$ que ligam, respectivamente, $a$ a $b$ e $c$ a $a$ sem cruzar $\Gamma$ ou $\Theta$ (e portanto, cruzando necessariamente $v$, que foi definido como segmento de reta aberto). Suponha que $l_{1}$ entra em $E$ antes de cruzar $v$ pela primeira vez. Se $l_{2}$ também entra em $E$ antes de cruzar $v$ pela primeira vez então ligamos esses dois trechos em $E$ e concluímos que $c$ pertence a mesma componente conexa de $a$. Senão, note que $l_{1}$ sai de $D$ depois de cruzar $v$ pela última vez, assim ligando esse trecho final de $l_{1}$ com $l_{2}$ vemos que $c$ pertence a mesma componente conexa de $b$. 
Vejamos agora que $(\Gamma \cup v \cup \Theta)^{C}$ possui ao menos duas componentes conexas, o que terminará de provar o desejado. Sejam $z \in E$ e $w \in D$. Suponha que $z$ e $w$ estão na mesma componente conexa de $(\Gamma \cup v \cup \Theta)^{C}$ e seja $\alpha$ uma curva conexa simples ligando $z$ a $w$ sem tocar $\Gamma \cup v \cup \Theta$.

Seja $\beta$ o segmento de reta que liga $z$ a $w$. Note que ele não cruza $\Gamma \cup \Theta$ por definição. Então $J:=\alpha \cup \beta$ é uma curva de Jordan que não intercepta $\Gamma \cup \Theta$. Veja então que um ponto extremo de $v$ pertence a int $(J)$. A componente conexa de $\omega\left(B_{0}\right)$ que passa por este extremo de $v$ é ilimitada e portanto precisa cruzar $\alpha$ ou $\beta$. Isso em qualquer caso leva a uma contradição.

Chamaremos de $\Omega_{1}$ e $\Omega_{2}$ as duas componentes conexas de $(\Gamma \cup v \cup \Theta)^{C}$. Note que $\Omega_{1}$ e $\Omega_{2}$ são abertas. O lema abaixo tem prova semelhante ao que fizemos anteriormente.

Lema 9. Seja $(p, q) \in \mathbb{Z}^{2}$. Para todo $w \in \mathbb{T}^{2}$ existe $z \in \pi^{-1}(w) \cap A$ e uma curva $\delta: \mathbb{R} \rightarrow \mathbb{R}^{2}$ que é limitada na horizontal, passa por todos os pontos da forma $z+(0, i)$, onde $i \in \mathbb{Z}$, e é tal que os conjuntos $\omega\left(B_{0}\right)$ e $\omega\left(B_{\pi}\right)+(p, q)$ estão em diferentes componentes conexas de $\delta^{C}$.

Demonstração. Como $\pi\left(\omega\left(B_{0}\right)\right) \cap \pi\left(\omega\left(B_{\pi}\right)\right)=\emptyset$ assuma, sem perda de generalidade, que $w \notin \pi\left(\omega\left(B_{0}\right)\right)$. Note então que existe um ponto $z \in$ $\pi^{-1}(w)$ e tal que $(z)_{1}>p$. Os pontos $z+(0, i)$ tem primeira coordenada estritamente maior que $p$ e portanto nenhum deles está em $A^{C}=\omega\left(B_{0}\right) \cup$ $\left(\omega\left(B_{\pi}\right)+(p, q)\right)$.

Pela proposição 34 sabemos que $z$ e $z+(0,1)$ estão na mesma componente conexa de $A^{C}$. Considere uma curva conexa $\gamma:[0,1] \rightarrow A^{C}$ tal que $\gamma(0)=z$ e $\gamma(1)=z+(0,1)$. A curva $\delta:=\cup_{i \in \mathbb{Z}}(\gamma+(0, i))$ satisfaz o pedido.

Corolário. Para todo $w \in \mathbb{T}^{2}$ existe $z \in \pi^{-1}(w) \cap \Omega_{1}$ e um inteiro $k$ tal que $z+(0, k) \in \pi^{-1}(w) \cap \Omega_{2}$.

Demonstração. Sejam $z$ e $\delta$ como no lema anterior. Se parametrizarmos a curva conexa $\delta$ por $\mathbb{R}$ (por consturção $\delta$ é união de curvas simples conexas de $z+(0, i)$ até $z+(0, i+1))$ podemos encontrar $T \in \mathbb{R}$ tal que $\delta(t) \cap v=\emptyset$ para todo $|t|>T$ (isso segue de que $\delta$ foi construída repetindo um mesmo segmento de comprimento finito). Considere $t_{1}<-T$ e $t_{2}>T$ tais que $\delta\left(t_{1}\right)=z_{j}$ e $\delta\left(t_{2}\right)=z_{k}$, onde $j, k \in \mathbb{N}$. Vamos mostrar que $z_{j}$ e $z_{k}$ não estão ambos em $\Omega_{i}$.

Suponha que estejam. Temos uma curva conexa $\alpha$ que liga $z_{j}$ com $z_{k}$ sem sair de $\Omega_{i}$. A curva $\left.\delta(]-\infty, t_{1}\right) \cup \alpha \cup \delta(] t_{2},+\infty[)$ é conexa, limitada na horizontal e ilimitada na vertical. Mas, por definição, essa curva não intercepta a curva conexa $\Gamma \cup v \cup \Theta$ : uma contradição, pois como não vale a teorema, $\Gamma \cup v \cup \Theta$ é ilimitada nas duas horizontais (pois $\Gamma$ é ilimitada na horizontal e pertence a $\omega\left(B_{0}\right)$, de forma que só tem que ser ilimitada para a "direita" e analogamente $\Theta$ é ilimitada para a "esquerda"). 
Corolário. $F\left(\Omega_{i}\right) \cap \Omega_{i} \neq \emptyset$ para $i=\{1,2\}$.

Demonstração. Como vimos anteriormente podemos assumir que $(0,0) \in$ $\rho(F)$. Pelo teorema de Franks existe $y \in \mathbb{T}^{2}$ fixos para $f$ e tal que $F(y)-y=$ $(0,0)$ para todo $\tilde{z} \in \pi^{-1}(y)$. Assim, pelo corolário anterior, concluímos que tanto $\Omega_{1}$ quanto $\Omega_{2}$ contém pontos fixos, o que prova o desejado.

Com isso obtemos a seguinte proposição.

Proposição 35. Ou $F\left(\overline{\Omega_{1}}\right) \subseteq \overline{\Omega_{1}}$ ou $F\left(\overline{\Omega_{2}}\right) \subseteq \overline{\Omega_{2}}$.

Demonstração. Pela definição de $v$ temos que $v \cap \omega\left(B_{0}\right)=\emptyset=v \cap\left(\omega\left(B_{\pi}\right)+\right.$ $\left.\left(p_{1}+p_{2}, q_{1}+q_{2}\right)\right)$. Como $\omega\left(B_{0}\right), \omega\left(B_{\pi}\right)$ são invariantes, $F(v) \cap \Gamma=\emptyset=$ $F(v) \cap \Theta$. Além disso $v$ foi tomada tal que $F(v) \cap v$. Assim,

$$
F(v) \cap(\Gamma \cup v \cup \Theta)=\emptyset
$$

Vamos assumir, sem perda de generalidade, que $F(v) \subseteq \Omega_{1}$.

Vamos mostrar que $F(\Gamma) \cap \Omega_{2}=\emptyset$. Observe que $F(\Gamma)$ é uma componente conexa de $\omega\left(B_{\pi}\right)$, pois $\Gamma$ é uma componente conexa de $\omega\left(B_{\pi}\right)$ (invariante). Assim, ou $F(\Gamma)=\Gamma$ ou $F(\Gamma) \cap \Gamma=\emptyset$.

Se $F(\Gamma)=\Gamma$ temos pela definição de $\Omega_{2}$ que $F(\Gamma) \cap \Omega_{2}=\emptyset$. Suponha então que $F(\Gamma) \cap \Gamma=\emptyset$ : É claro que $F(\Gamma) \cap \partial \Omega_{1}=\emptyset$ pois $F(\Gamma) \cap v=\emptyset \mathrm{e}$ $F(\Gamma) \cap \omega\left(B_{0}\right)=\emptyset$. Como $\Gamma \cup v$ é conexo e $F(v) \subseteq \Omega_{1}$ então $F(\Gamma) \subseteq \Omega_{1}$ e portanto $F(\Gamma) \cap \Omega_{2}=\emptyset$. Analogamente concluímos que $F(\Theta) \cap \Omega_{2}=\emptyset$.

Assim, como $F(v) \subseteq \Omega_{1}$, temos necessariamente que $F\left(\partial \Omega_{1}\right) \cap \Omega_{2}=\emptyset$. Mas $\Omega_{1}$ e $\Omega_{2}$ são conexos, de forma que $\Omega_{2} \subseteq F\left(\Omega_{1}\right)$ ou $\Omega_{2} \cap F\left(\Omega_{1}\right)=\emptyset$.

Suponha que $\Omega_{2} \subseteq F\left(\Omega_{1}\right)$. Teríamos que $F^{-1}\left(\Omega_{2}\right) \subseteq \Omega_{1}$, de forma que $F^{-1}\left(\Omega_{2}\right) \cap \Omega_{2}=\emptyset$. Mas pelo corolário anterior $F\left(\Omega_{2}\right) \cap \Omega_{2} \neq \emptyset$, uma contradição. Vemos assim que necessariamente $\Omega_{2} \cap F\left(\Omega_{1}\right)=\emptyset$ o que implica $F\left(\overline{\Omega_{1}}\right) \subseteq \overline{\Omega_{1}}$.

Finalmente chegamos ao

Proposição 36. Se $\pi\left(\omega\left(B_{0}\right)\right) \cap \pi\left(\omega\left(B_{\pi}\right)\right)=\emptyset$ então vale o teorema.

Demonstração. Suponha que não vale o teorema. Pela proposição anterior, ou $F\left(\overline{\Omega_{1}}\right) \subseteq \overline{\Omega_{1}}$ ou $F\left(\overline{\Omega_{2}}\right) \subseteq \overline{\Omega_{2}}$. Suponha que vale o primeiro caso (o outro caso é análogo). Vemos facilmente por indução que $F^{k}\left(\overline{\Omega_{1}}\right) \subseteq \overline{\Omega_{1}}$ para $k \in \mathbb{N}$. Como vimos no início, podemos assumir que $(0,1)$ e $(0,-1) \in \rho(F)$. Pelo teorema de Franks existem $w_{1}, w_{2} \in \mathbb{T}^{2}$ fixos para $f$ e tais que

$$
F^{k}\left(z_{1}\right)-z_{1}=k(0,1) \quad \text { e } \quad F^{k}\left(z_{2}\right)-z_{2}=k(0,-1)
$$

para todo $z_{1} \in \pi^{-1}\left(w_{1}\right), z_{2} \in \pi^{-1}\left(w_{2}\right)$ e $k \in \mathbb{Z}$. Pelo lema 9 e seu primeiro corolário, podemos assumir que $z_{1} \in \Omega_{1} \cap \pi^{-1}\left(w_{1}\right), z_{2} \in \Omega_{1} \cap \pi^{-1}\left(w_{2}\right)$ e que existem curvas $\gamma_{1}$ e $\gamma_{2}$ passando respectivamente por todos os pontos 
da forma $z_{1}+(0, k)$ e $z_{2}+(0, k)$ para $k \in \mathbb{Z}$ que são limitadas na horizontal, ilimitadas na vertical e não interceptam $\Gamma$ ou $\Theta$.

Suponha que para $i \in\{1,2\}$ e todo $k>0$ temos que $z_{i}+k(0,1)=$ $F^{k}\left(z_{i}\right) \in \Omega_{1}$. O trecho $\delta_{1}$ de $\gamma_{1}$ que liga $z_{1}$ com $z_{1}+(0, k)$ para todo $k>0$ pode eventualmente cruzar para $\Omega_{2}$, mas para isso precisa cruzar o segmento $v$ (pois $\gamma_{1}$ não cruza $\Gamma$ ou $\Theta$ ). No entanto ele só pode cruzar $v$ um número finito de vezes. Como estamos supondo que $F\left(\overline{\Omega_{1}}\right) \subseteq \overline{\Omega_{1}}$, se o segmento de $\delta_{1}$ que liga $z_{1}+(0, k)$ com $z_{1}+(0, k+1)$ (para $\left.k>0\right)$ cruzar $v$, podemos trocar ele por $F\left(\gamma_{0}\right)$, onde $\gamma_{0}$ é o trecho de $\delta_{1}$ que liga $z_{1}$ com $z_{1}+(0,1)$. Obtemos então uma curva $\tilde{\gamma_{1}} \subseteq \Omega_{1}$ que é ilimitada para cima e limitada na horizontal (já que só fizemos um número finito de trocas na curva $\delta_{1}$ que era limitada na horizontal).

Analogamente construimos uma curva $\tilde{\gamma_{2}} \subseteq \Omega_{1}$ que liga $z_{2}$ com $z_{2}+$ $(0,-k)$ para todo $k>0$ é ilimitada para baixo e limitada na horizontal. Finalmente, como pedimos que $z_{1}, z_{2} \in \Omega_{1}$, podemos ligar $\tilde{\gamma_{1}}$ com $\tilde{\gamma_{2}}$ obtendo uma curva $\tilde{\delta}$ ilimitada na vertical (para cima e para baixo) e limitada na horizontal totalmente contida em $\Omega_{1}$ : mas isso é uma contradição, pois $\tilde{\delta}$ precisa interceptar $\Gamma \cup v \cup \Theta$.

Resta então admitir que para $i \in\{1,2\}$ e algum $k>0$ valer que $z_{i}+$ $k(0,1)=F^{k}\left(z_{i}\right) \in \Omega_{2}$. Mas isso também leva a uma contradição pois $F^{k}\left(\overline{\Omega_{1}}\right) \subseteq \overline{\Omega_{1}}$ e $\Omega_{1} \cap \Omega_{2}=\emptyset$. 


\section{Capítulo 7}

\section{Comentários finais}

Podemos notar que a irracionalidade de $\alpha$ (a segunda coordenada do vetor de rotação médio) só foi usada apenas em três ocasiões (juntamente com o lema de Atkinson): Na prova da proposição 20 na página 28, do teorema 29 na página 39 e na prova da proposição 31 na página 41.

Nessas três instâncias é possível provar os mesmos resultados se assumirmos, uma hipótese alternativa que explicamos a seguir. Como $\rho(F)$ é um segmento vertical contido em $\{0\} \times \mathbb{R}$, se $z \in \mathbb{T}^{2}$ é periódico de período $n \in \mathbb{N}$ e $x \in \pi^{-1}(z)$ então $F^{n}(x)=x+(0, i)$ para algum $i \in \mathbb{Z}$. Queremos não exatamente que esses pontos sejam densos em $\mathbb{R}^{2}$, mas que em toda vizinhança em torno de qualquer $x \in \mathbb{R}^{2}$ exista $y$ com $\frac{i(x)}{n(x)} \neq \frac{i(y)}{n(y)}$ (isso fará com que diferentes componentes conexas se interceptem, o que não era permitido nas demonstrações que apresentamos). É claro que se existe um conjunto denso composto por pontos pontos periódicos com vetores de rotação dois-a-dois distintos teremos o desejado.

Como supomos que a medida de Lebesgue é preservada o teorema de Franks garante a existência de ao menos um ponto periódico para cada vetor de $\rho(F)$ com segunda racional, descreveremos a hipótese alternativa dizendo que "os pontos dados como no teorema de Franks são densos em $\mathbb{R}^{2}$.

Pergunta. Quando F possui vetor de rotação médio racional a ergodicidade implica que os pontos dados como no teorema de Franks são densos em $\mathbb{R}^{2}$ ?

A ergodicidade com respeito a medida de Lebesgue diz, por exemplo, que a órbita de quase todo ponto é densa no toro. Além disso, como os conjuntos $\omega\left(B_{0}\right)$ e $\omega\left(B_{\pi}\right)$ são totalmente invariantes, nosso contexto e as propriedades de $\rho(F)$ nos dizem que podemos considerar apenas o caso em que $\rho_{\lambda}(F)=(0,0)$.

Ao invés de tentar resolver a pergunta acima podemos pensarem se há uma prova direta quando os pontos como no teorema de Franks não são densos (o que possivelmente eliminaria a necessidade de olhar para o vetor 
de rotação médio). Até agora não foi possível obter uma prova baseado nessas idéias.

Finalmente, notamos que também que podemos aplicar o teorema aqui provado para mostrar o resultado no caso em que o conjunto de rotação é um segmento de inclinação racional que passa por um ponto de $\mathbb{Q}^{2}$.

Para ver isso observe que se nesse caso existe uma matriz de rotação com coordenadas inteiras $A \in G L(2, \mathbb{Z})$ tal que $A \rho(F)$ é um segmento contido em $\{0\} \times \mathbb{R}$. De fato, se $\rho(F)$ tem inclinação racional $p / q$ podemos encontrar $x, y \in \mathbb{Z}$ tais que $p x+q y=1$ e fazer

$$
A=\left(\begin{array}{cc}
p & -q \\
y & x
\end{array}\right)
$$

Observamos que $\operatorname{det}(A)=1$ e que $A(0,0)=0$ e $A(q, p)=(0,1)$, de forma que $A \rho(F)$ precisa ser um segmento contido em $\{0\} \times \mathbb{R}$. Temos então o seguinte resultado

Lema 10 ([KK08]). Seja $f$ um homeomorfismo de $\mathbb{T}^{2}$ homotópico a identidade e $F$ um levantamento de $f$. Seja $A \in G L(2, \mathbb{Z})$ e $h$ um homeomorfismo de $\mathbb{T}^{2}$ homotópico a A com levantamento $H$. Então

$$
\rho\left(H F H^{-1}\right)=A \rho(F)
$$

Em particular $\rho\left(A F A^{-1}\right)=A \rho(F)$.

Podemos então aplicar o resultado aqui provado para $g=h f h^{-1}$ (que vemos facilmente ser homotópica a identidade e ergódica desde $f$ o seja). Concluímos que existe levantamento $G$ de $g$ e $m \in \mathbb{N}$ tal que o deslocamento de $G^{m}$ é limitado na horizontal, de forma que o deslocamento de $F^{m}$ é limitado na direção de ângulo $p / q$ (a inclinação de $\rho(F)$ ).

Dizemos, como em [KT12b], que um homeomorfismo de $\mathbb{T}^{2}$ homotópico a identidade é anular se existe $M>0, v \in \mathbb{Z}_{*}^{2}$ e um levantamento $G$ de $f$ tal que

$$
-M \leq\left\langle G^{n}(x)-x, \frac{v}{\|v\|}\right\rangle \leq M, \quad \text { para todo } x \in \mathbb{R}^{2} \text { e todo } n \in \mathbb{Z}
$$

Em particular, o teorema provado aqui diz que, se $f: \mathbb{T}^{2} \rightarrow \mathbb{T}^{2}$ é um homeomorfismo homotópico a identidade e $F: \mathbb{R}^{2} \rightarrow \mathbb{R}^{2}$ um levantamento de $f$ tal que $\rho(F)$ é um segmento não degenerado de inclinação racional que passa por um ponto de $\mathbb{Q}^{2}$ e, se além disso, $f$ é ergódico com respeito a medida de Lebesgue no toro e seu vetor de rotação médio é da forma $(0, \alpha)$ para $\alpha \in \mathbb{R} \backslash \mathbb{Q}$ então alguma potência de $f$ é anular. Isto, dentro de um contexto adequado (veja [KT12b]) mostra que os homeomorfismos como acima não possuem comportamento estritamente toral, podendo em ser entendidos, no sentido adequado, como homeomorfismos do anel. 


\section{Referências Bibliográficas}

[AP00] S. Alpern and V.S. Prasad. Typical dynamics of volume preserving homeomorphisms. Number 139. Cambridge Univ Pr, 2000.

[Atk76] G. Atkinson. Recurrence of co-cycles and random walks. Journal of the London Mathematical Society, 2(3):486, 1976.

[AZT11] S. Addas-Zanata and F.A. Tal. Homeomorphisms of the annulus with a transitive lift. Mathematische Zeitschrift, 267(3):971-980, 2011.

[BCRP04] F. Béguin, S. Crovisier, F.L. Roux, and A. Patou. Pseudorotations of the closed annulus: variation on a theorem of $\mathrm{j}$ kwapisz. Nonlinearity, 17:1427, 2004.

[Beg07] F. Beguin. Ensembles de rotations des homéomorphismes du tore $\mathbb{T}^{2}$. Notas de Aula, 2007.

[BLR07] F. Beguin and F. Le Roux. Dynamique topologique sur les surfaces. Notas de Aula, 2007.

[BT12] R.B. Bortolatto and F.A. Tal. Ergodicity and annular homeomorphisms of the torus. Arxiv preprint arXiv:1201.5803, 2012.

[Dáv11] P. Dávalos. On torus homeomorphisms whose rotation set is an interval. Arxiv preprint arXiv:1111.2378, 2011.

[FM90] J. Franks and M. Misiurewicz. Rotation sets of toral flows. In Proc. Amer. Math. Soc, volume 109, page 243, 1990.

[Fra88] J. Franks. Recurrence and fixed points of surface homeomorphisms. Ergodic theory and dynamical systems, 8(8):99-107, 1988.

[Fra89] J. Franks. Realizing rotation vectors for torus homeomorphisms. Trans. Amer. Math. Soc, 311(1):107-115, 1989.

[Fra96] J. Franks. The rotation set and periodic points for torus homeomorphisms. Arxiv preprint math/9605228, 1996. 
[GTAZ11] B. Garcia, F.A. Tal, and S. Addas-Zanata. Dynamics of homeomorphisms of the torus homotopic to dehn twists. Arxiv preprint arXiv:1111.5561, 2011.

[Jäg08] T. Jäger. The concept of bounded mean motion for toral homeomorphisms. Arxiv preprint arXiv:0802.4187, 2008.

[Jäg09] T. Jäger. Linearization of conservative toral homeomorphisms. Inventiones Mathematicae, 176(3):601-616, 2009.

[JZ98] Leo B. Jonker and Lei Zhang. Torus homeomorphisms whose rotation sets have empty interior. Ergodic Theory and Dynamical Systems, 18(05):1173-1185, 1998.

[KH96] A. Katok and B. Hasselblatt. Introduction to the modern theory of dynamical systems, volume 54. Cambridge Univ Pr, 1996.

[KK08] A. Kocsard and A. Koropecki. Free curves and periodic points for torus homeomorphisms. Ergodic Theory Dynam. Systems, 28:1895-1915, 2008.

[KT12a] A. Koropecki and F.A. Tal. Area-preserving irrotational diffeomorphisms of the torus with sublinear diffusion. Arxiv preprint arXiv:1206.2409, 2012.

[KT12b] A. Koropecki and F.A. Tal. Strictly toral dynamics. Arxiv preprint arXiv:1201.1168, 2012.

[Kwa92] J. Kwapisz. Every convex polygon with rational vertices is a rotation set. Ergodic Theory and Dynamical Systems, 12(02):333339, 1992.

[Kwa95] J. Kwapisz. A toral diffeomorphism with a nonpolygonal rotation set. Nonlinearity, 8:461, 1995.

[LC05] P. Le Calvez. Une version feuilletée équivariante du théoreme de translation de brouwer. Publications Mathématiques de l'IHÉS, 102(1):1-98, 2005.

[LM91] J. Llibre and R. MacKay. Rotation vectors and entropy for homeomorphisms of the torus isotopic to the identity. Ergodic Theory Dynam. Systems, 11(1):115-128, 1991.

[MZ89] M. Misiurewicz and K. Ziemian. Rotation sets for maps of tori. Journal of the London Mathematical Society, 2(3):490, 1989.

[MZ91] M. Misiurewicz and K. Ziemian. Rotation sets and ergodic measures for torus homeomorphisms. Fund. Math, 137(1):45-52, 1991. 
[New39] M.H.A. Newman. Elements of the topology of plane sets of points. Bull. Amer. Math. Soc. 45 (1939), 822-824. DOI: 10.1090/S0002-9904-1939-07087-0 PII: S, 2(9904):070870,1939 .

[NPT83] S. Newhouse, J. Palis, and F. Takens. Bifurcations and stability of families of diffeomorphisms. Publications Mathématiques de l'IHÉS, 57(1):5-71, 1983.

[Tal12] F.A. Tal. Transitivity and rotation sets with nonempty interior for homeomorphisms of the 2-torus. Proc. Am. Math. Soc., 140:3567-3579, 2012. 